\title{
SEMIABELIAN VARIETIES OVER SEPARABLY CLOSED FIELDS, MAXIMAL DIVISIBLE SUBGROUPS, AND EXACT SEQUENCES
}

\author{
FRANCK BENOIST ${ }^{1}$, ELISABETH BOUSCAREN ${ }^{2}$ AND ANAND PILLAY ${ }^{3}$ \\ ${ }^{1}$ Department of Mathematics, Univ. Paris-Sud, Bat. 425, F-91405 Orsay Cedex, \\ France (franck.benoist@math.u-psud.fr) \\ ${ }^{2}$ Department of Mathematics, CNRS - Univ. Paris-Sud, Bat. 425, \\ F-91405 Orsay Cedex, France (elisabeth.bouscaren@math.u-psud.fr) \\ ${ }^{3}$ Department of Mathematics, University of Notre Dame, 281 Hurley Hall, \\ Notre Dame, IN 46556, USA (apillay@nd.edu)
}

(Received 22 May 2014; revised 23 June 2014; accepted 24 June 2014; first published online 17 July 2014)

\begin{abstract}
Given a separably closed field $K$ of characteristic $p>0$ and finite degree of imperfection, we study the $\sharp$ functor which takes a semiabelian variety $G$ over $K$ to the maximal divisible subgroup of $G(K)$. Our main result is an example where $G^{\sharp}$, as a 'type-definable group' in $K$, does not have 'relative Morley rank', yielding a counterexample to a claim in Hrushovski [J. Amer. Math. Soc. 9 (1996), 667-690]. Our methods involve studying the question of the preservation of exact sequences by the $\sharp$ functor, and relating this to issues of descent as well as model-theoretic properties of $G^{\sharp}$. We mention some characteristic 0 analogues of these 'exactness-descent' results, where differential algebraic methods are more prominent. We also develop the notion of an iterative D-structure on a group scheme over an iterative Hasse field, which is interesting in its own right, as well as providing a uniform treatment of the characteristic 0 and characteristic $p$ cases of 'exactness descent'.
\end{abstract}

\section{Introduction}

For a semiabelian variety $G$ over a separably closed field $K$ of characteristic $p>0$ and finite degree of imperfection, the group $p^{\infty} G(K)=\bigcap_{n} p^{n}(G(K))$ played a big role in Hrushovski's proof of the function field Mordell-Lang conjecture in positive characteristic [16]. The group $p^{\infty} G(K)$, which we also sometimes call $G^{\sharp}$, is type definable in the structure $(K,+, \cdot)$. It was claimed in [16] (in the Remark just before Lemma 2.15) that $p^{\infty} G(K)$ always has finite relative Morley rank. One of the reasons or motivations for writing the current paper is to show that this is not the case: there are $G$ such that $p^{\infty} G(K)$ does not even have relative Morley rank. (Note that, however, Lemma 2.15 itself does hold; the generic type of $p^{\infty} G(K)$ is indeed 'thin', which implies that $p^{\infty} G(K)$ does

The first author was supported by a Post Doctoral position of the Marie Curie European Network MRTN CT-2004-512234 (MODNET) (Leeds, 2007-2008). The first author and the second author were partially supported by ANR MODIG (ANR-09-BLAN-0047) Model theory and Interactions with Geometry. The third author was supported by a Marie Curie Excellence Chair 024052, and EPSRC grants EP/F009712/1 and EP/I002294/1. 
have finite $U$-rank, but just not that it has finite relative Morley rank. The finiteness of $U$-rank suffices for all the results in $\S 4$ of [16], in particular Proposition 4.3, to go through, and hence the validity of the main results of [16] is unaffected.) Hrushovski used expressions such as 'Morley dimension' or 'internal Morley dimension' for what we call here relative Morley rank. The notion is somewhat subtle and concerns performing a Cantor-Bendixon analysis inside a closed space of types. Details and examples are given in $\S 2.3$.

As the second author noticed some time ago, the 'relative Morley rank' problem is related in various ways to whether the $p^{\infty}$ (or $\sharp$ ) functor preserves exact sequences. So another theme of the current paper is to give conditions on an exact sequence $0 \rightarrow G_{1} \rightarrow$ $G_{2} \rightarrow G_{3} \rightarrow 0$ of semiabelian varieties over $K$ which imply exactness of the sequence $0 \rightarrow$ $G_{1}^{\sharp} \rightarrow G_{2}^{\sharp} \rightarrow G_{3}^{\sharp} \rightarrow 0$, as well as giving situations where the sequence of $G_{i}^{\sharp}$ is NOT exact.

A third theme relates the preservation of exactness by $\sharp$ to the issue of descent of a semiabelian variety $G$ over $K$ to the field of 'constants' $K^{p^{\infty}}=\bigcap_{n} K^{p^{n}}$ of $K$.

If $K$ has degree of imperfection $e$ (meaning that $K$ has dimension $p^{e}$ as a vector space over its $p$ th powers $K^{p}$ ), then $K$ can be equipped naturally with $e$ commuting iterative Hasse derivations. We will, for simplicity, mainly consider the case where $e=1$ (so, for example, where $\left.K=\mathbb{F}_{p}(t)^{\mathrm{sep}}\right)$, in which case we have a single iterative Hasse derivation $\left(\partial_{n}\right)_{n}$ whose field of absolute constants is $K^{p^{\infty}}$. This differential structure on $K$ will play a role in some proofs, by virtue of so-called D-structures on varieties over $K$.

The analogue in characteristic 0 of the differential field $\left(K,\left(\partial_{n}\right)_{n}\right)$ is simply a differentially closed field $(K, \partial)$ (of characteristic zero). And for an abelian variety $G$ over our characteristic 0 differentially closed field $K$, we have what is often called the 'Manin kernel' for $G$, the smallest Zariski-dense 'differential algebraic' subgroup of $G(K)$, which we denote again by $G^{\sharp}$. The issues of preservation of exactness by $\sharp$ and the relationship to descent to the field $\mathcal{C}$ of constants make sense in characteristic 0 too.

In characteristic $p$, it is possible to obtain our results with a purely algebraic approach using $p$-torsion and Tate modules (carried out in $\S 4$ ). In characteristic 0 , we need to use differential algebraic methods, in particular D-structures. But in fact the algebraic proofs given in characteristic $p$ can also be seen as involving D-structures, and we take the opportunity of giving such a uniform proof in all characteristics in $\S 5$.

Our paper builds on earlier work by the second author and Françoise Delon [8], where, among other things, the groups $G^{\sharp}$ (in positive characteristic) are characterized as precisely the commutative divisible type-definable groups in separably closed fields. Our results, especially in characteristic 0 , are also influenced by and closely related to themes in the third author's joint paper with Daniel Bertrand [6].

Let us now describe the content and results of the paper.

Section 2 recalls key notions and facts about differential fields, and semiabelian varieties over separably closed fields. We also discuss relative Morley rank, preservation of descent under isogeny, and some properties of $p^{\infty} G(K)$.

In $\S 3$, we introduce the $\sharp$ functor in all characteristics, and begin relating relative Morley rank to exactness.

Section 4 concentrates on the characteristic $p$ case. We begin by making some observations about descent of semiabelian varieties and Tate modules, proving for 
example that an ordinary semiabelian variety $G$ descends to the constants of $K$ if and only if all of the (power of $p$ ) torsion of $G$ is $K$-rational ( $\S 4.1$ ). We make no claim that our results on descent are especially novel, and we would not be surprised if they were explicit or implicit in the literature on semiabelian varieties in positive characteristic. However, we were unable to find precise references despite consulting several experts. In $\S 4.2$, we answer the original question which motivated this paper. In Proposition 4.12, we show that, if $0 \rightarrow G_{1} \rightarrow G_{2} \rightarrow G_{3} \rightarrow 0$ is an exact sequence of ordinary semiabelian varieties such that $G_{1}$ and $G_{3}$ descend to the constants, $\mathcal{C}$, then the sequence of the $G_{i}^{\sharp}$ is exact if and only if $G_{2}$ descends to $\mathcal{C}$. This yields an example of a semiabelian variety $G$ such that $G^{\sharp}$ does not have relative Morley rank (in fact, the example is simply any nonconstant extension of a constant ordinary abelian variety by an algebraic torus). See Corollary 4.14, which as mentioned above is among the main results of our paper. The remainder of $\S 4$ contains both positive and negative results about preservation of exactness by $\sharp$ in various situations. In particular, we give an example of an exact sequence of ordinary abelian varieties for which the $\sharp$ functor does not preserve exactness. This cannot happen in characteristic 0, as shown in the next section.

In $\S 5$, we switch to differential algebraic methods in order to treat uniformly both characteristic 0 and characteristic $p$. In $\S 5.1$, we recall the definition of D-structures for group schemes and the fact that a semiabelian variety $G$ over a Hasse field $K$ descends to the constants of $K$ if and only if $G$ admits an iterative D-structure.

In order to relate exactness of the $\sharp$ functor and descent in characteristic 0 , we use, as in [6], the universal extension $\tilde{G}$ of $G$ by a vector group, which always admits a unique D-structure. In characteristic $p$, we need to replace this universal extension by a ( $p$-divisible) proalgebraic group, also called $\tilde{G}$. In $\S 5.2$, in characteristic $p$, we endow $\tilde{G}$ with an iterative D-structure, and prove the characteristic $p$ version of the characteristic 0 results relating descent and the D-structure on $\tilde{G}$. Finally, in $\S 5.3$, we can then give a uniform proof, in all characteristics (Proposition 5.21), of the fundamental result (Proposition 4.12) proved previously in characteristic $p$.

We should say that, as far as 'algebraic geometry' is concerned, this paper is elementary, and, even in $\S 5.3$, it does not make heavy use of modern methods. The reader is referred to [12] for a modern scheme-theoretic treatment of descent, $K / k$-trace, etc., for abelian varieties in positive characteristic. As is pointed out there, much of the literature on such questions, and on important results such as the Lang-Neron theorem, remains in the language of Weil. The same will be to some extent true of the current paper, where our real aim and motivation is to understand $G(K)$ as a definable group in the structure $(K,+, \cdot)$, as well as its type-definable subgroups.

\section{Preliminaries}

\subsection{Hasse fields}

We summarize here basic facts and notation about the fields $K$ that concern us. More details can be found in $[4,41]$ for the characteristic $p$ case and [21] for the characteristic 0 case. 
If $K$ is a separably closed field of characteristic $p>0$, then the dimension of $K$ as a vector space over the field $K^{p}$ of $p$ th powers is infinite or a power $p^{e}$ of $p$. In the second case, $e$ is called the degree of imperfection (we will just say the 'invariant') of $K$, and we will be interested in the case when $e \geqslant 1$ (and often when $e=1$ ). For $e$ finite, a $p$-basis of $K$ is a set $a_{1}, \ldots, a_{e}$ of elements of $K$ such that $\left\{a_{1}^{n_{1}} a_{2}^{n_{2}} \ldots a_{e}^{n_{e}}: 0 \leqslant n_{i}<p^{e}\right\}$ form a basis of $K$ over $K^{p}$.

The first-order theory of separably closed fields of characteristic $p>0$ and invariant $e$ (in the language of rings) is complete (and model complete). We call the theory $\mathrm{SCF}_{p, e}$. It is also stable (but not superstable), and certain natural (inessential) expansions that we mention below have quantifier elimination.

For $R$ an arbitrary ring (commutative with a 1), an iterative Hasse derivation $\partial$ on $R$ is a sequence $\left(\partial_{n}: n=0,1, \ldots\right)$ of additive maps from $R$ to $R$ such that

(i) $\partial_{0}$ is the identity,

(ii) for each $n, \partial_{n}(x y)=\sum_{i+j=n} \partial_{i}(x) \partial_{j}(y)$, and,

(iii) for all $i, j, \partial_{i} \circ \partial_{j}=\left(\begin{array}{c}i+j \\ i\end{array}\right) \partial_{i+j}$ (iterativity).

Note that $\partial_{1}$ is a derivation, and that, when $R$ has characteristic $0, \partial_{n}=\partial_{1}^{n} / n$ !. (So, in the characteristic 0 case, the whole sequence $\left(\partial_{n}\right)_{n}$ is determined by $\partial_{1}$.)

In some rare cases, we will speak about noniterative Hasse derivation, meaning that the third condition is not required.

By the constants of $\left(R,\left(\partial_{n}\right)_{n} \geqslant 0\right)$, one usually means $\left\{r \in R: \partial_{1}(r)=0\right\}$, and by the absolute constants $\left\{r \in R: \partial_{n}(r)=0\right.$ for all $\left.n>0\right\}$. In this paper, we will mainly consider the field of absolute constants, denoted $\mathcal{C}$, and refer to them in what follows as 'the constants'.

If $\partial^{1}$ and $\partial^{2}$ are iterative Hasse derivations on $R$, we say that they commute if each $\partial_{i}^{1}$ commutes with each $\partial_{j}^{2}$.

Fact 2.1. (i) If $K$ is a separably closed field of invariant $e \geqslant 1$, then there are commuting iterative Hasse derivations $\partial^{1}, \ldots, \partial^{e}$ on $K$ such that the common constant of $\partial_{1}^{1}, \ldots, \partial_{1}^{e}$ is $K^{p}$. In this case, the common (absolute) constant of $\partial^{1}, \ldots, \partial^{e}$ is the field $K^{p^{\infty}}=\bigcap_{n} K^{p^{n}}$.

(ii) Moreover, in (i), if $a_{1}, \ldots, a_{e}$ is a p-basis of $K$, then each $\partial_{j}^{i}$ is definable in the field $K$ over parameters consisting of the $a_{1}, \ldots, a_{e}$ and their images under the maps $\partial_{m}^{n}(n=1, \ldots, e, m \geqslant 0)$.

(iii) The theory $\mathrm{CHF}_{p, e}$ of separably closed fields of degree e, equipped with e commuting iterative Hasse derivations $\partial^{1}, \ldots, \partial^{e}$, whose common field of constants is $K^{p}$, is complete and stable, with quantifier elimination (in the language of rings together with unary function symbols for each $\left.\partial_{n}^{i}, i=1, \ldots, e, n>0\right)$.

Note that, after adding names for a $p$-basis $a_{1}, \ldots, a_{e}$ of the separably closed field $K$, we obtain for each $n$ a basis $1, d_{1}, \ldots, d_{p^{n}-1}$ of $K$ over $K^{p^{n}}$, and the functions $\lambda_{n, i}$ such that $x=\sum_{i}\left(\lambda_{n, i}(x)\right)^{p^{n}} d_{i}$ for all $x$ in $K$, are definable with parameters $a_{1}, \ldots, a_{e}$ in the field $K$. The theory of separably closed fields also has quantifier elimination in the language with symbols for a $p$-basis and for each $\lambda_{n, i}$. The relation between the $\lambda$-functions and the $\partial_{j}^{i}$ is given in $\S 2$ of [4]. 
In the current paper, we concentrate on the iterative Hasse derivation formalism. In fact, when we mention separably closed fields $K$ with an iterative Hasse structure, we will usually assume that $e=1$, and so $K$ is equipped with a single iterative Hasse derivation $\partial=\left(\partial_{n}\right)_{n}$. The basic example is $\mathbb{F}_{p}(t)^{\mathrm{sep}}$ (where ${ }^{\text {sep }}$ denotes separable closure) with $\partial_{1}(t)=$ 1 and $\partial_{i}(t)=0$ for all $i>1$. The assumption that $e=1$ is made here for the sake of simplicity, as some of the results we will be quoting are only explicitly written out for this case, but it will be no real restriction, thanks to the following.

Fact 2.2 (See, for example, [4]). Let $K_{0}$ be an algebraically closed field of characteristic $p$, and let $K_{1}$ be a finitely generated extension of $K_{0}$. Then there is a separably closed field $K$ of degree of imperfection 1 , extending $K_{1}$ and such that $K_{0}=K^{p^{\infty}}$.

Our characteristic 0 analogue is simply a differentially closed field $(K, \partial)$ of characteristic 0 , where now $\partial$ is the single distinguished derivation (rather than a sequence). The corresponding first-order theory is $\mathrm{DCF}_{0}$, in the language of rings together with a symbol for $\partial$. The theory $\mathrm{DCF}_{0}$ is complete with quantifier elimination, but is now $\omega$-stable.

\subsection{Varieties, semiabelian varieties, and separable morphisms}

From now on, $K$ is an algebraically closed field of characteristic 0 , or a separably closed field of characteristic $p$ and of finite degree of imperfection $e \geqslant 1$, and $\bar{K}$ denotes an algebraic closure of $K$.

As already mentioned in the introduction, we will use mainly Weil type language in this paper, except in $\S 5.2$. A variety over $K$, or defined over $K$, will always be a separated reduced scheme of finite type over $K$. We denote by $V(L)$ the set of $L$-rational points of $V$, for $L$ an extension of $K$. Recall that, when $K$ is separably closed, and $V$ is over $K, V(K)$ is Zariski dense in $V$. We will often identify $V$ with its set of geometric points $V(\bar{K})$. For $L$ an extension of $K$, we will denote $V_{L}=V \times_{K} L$ (extension of scalars or base change).

Recall that, if $V$ and $W$ are two irreducible varieties over $K$, and $f$ is a dominant $K$-morphism from $V$ to $W, f$ is said to be separable if the function field extension $K(W) \subset$ $K(V)$ is separable.

The following is classical. For the convenience of the reader, we include a short (model-theoretic) proof in Appendix A.

Fact 2.3. Let $G, H$ be two connected algebraic groups defined over $K$, and let $f$ be a dominant separable homomorphism from $G$ to $H$ (equivalently a surjective separable homomorphism from $G(\bar{K})$ onto $H(\bar{K})$ ). Then $f$ takes $G(K)$ surjectively onto $H(K)$.

In this paper, we will only consider exact sequences of algebraic groups

$$
0 \rightarrow G_{1} \stackrel{g}{\rightarrow} G_{2} \stackrel{f}{\rightarrow} G_{3} \rightarrow 0
$$

such that both morphisms are separable. These are sometimes called strict exact sequences [34]. We will say also that $G_{2}$ is an algebraic group extension of $G_{3}$ by $G_{1}$, denoted by $G_{2} \in \operatorname{EXT}\left(G_{3}, G_{1}\right)$. By the assumption of separability of the morphisms, 
$G_{3}$ is isomorphic (as an algebraic group) to $G_{2} / g\left(G_{1}\right)$, and $G_{1}$ is isomorphic to a closed subgroup of $G_{2}$.

We will say that the exact sequence is over $K$ if the groups $G_{1}, G_{2}, G_{3}$ are algebraic groups over $K$ and $f, g$ are separable $K$-morphisms of algebraic groups.

We now recall some very basic facts about semiabelian varieties. We will be particularly interested in rationality issues, that is, in the groups of $K$-rational points of some basic subgroups of $G(K)$. There are many classical references for abelian varieties (for example [26], or [19]). For the case of tori, see, for example, [9].

It is then easy to obtain the corresponding facts for the case of arbitrary semiabelian varieties.

Recall that a semiabelian variety $G$ (over $K$ ) is an extension of an abelian variety by a torus, i.e.,

$$
0 \rightarrow T \rightarrow G \rightarrow A \rightarrow 0
$$

where $T$ is a torus over $K, A$ is an abelian variety over $K$, and the two morphisms are separable $K$-morphisms ( $G$ is then also an algebraic group over $K$ ).

The following facts hold when $K$ is separably closed.

Fact 2.4. (i) Let $T$ be a torus over $K$. Then $T$ is $K$-split; i.e., $T$ is isomorphic over $K$ to some product of the multiplicative group, $\left(\mathbb{G}_{m}\right)^{\times n}$. Any closed subgroup of $T_{\bar{K}}$ is then also defined over $K$.

(ii) Semiabelian varieties are commutative and divisible; i.e., $G(\bar{K})$, the group of $\bar{K}$-rational points of $G$, is a commutative divisible group.

(iii) Let $G$ be a semiabelian variety over $K$. Then any closed connected subgroup of $G_{\bar{K}}$ is defined over $K$.

Definition 2.5. Let $K_{0} \subset K_{1}$ be an extension of fields, and let $G$ be an algebraic group over $K_{1}$. We will say that $G$ descends to $K_{0}$ if $G$ is isomorphic to $H_{K_{1}}$ for some algebraic group $H$ over $K_{0}$.

As semiabelian varieties are defined as extensions, one should check what descent exactly means in that case. The following fact, which follows from classical manipulations on $\operatorname{EXT}(A, T)$ (see, for example, [34]), deals with this question.

Fact 2.6. Let $K_{0} \subset K_{1}$ be separably closed fields, and let $G$ be a semiabelian variety defined over $K_{1}$, which is an extension of $A$ by $T=\left(\mathbb{G}_{m}^{n}\right)_{K_{1}}$. If $G$ descends to $K_{0}$, i.e., if $G$ is isomorphic to $\left(G_{0}\right)_{K_{1}}$ for some semiabelian variety $G_{0}$ over $K_{0}$, then we have the following:

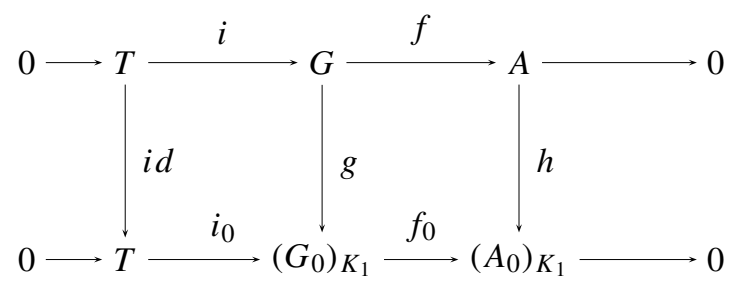


where $g$ and $h$ are isomorphisms, and $0 \rightarrow\left(\mathbb{G}_{m}^{n}\right)_{K_{0}} \rightarrow G_{0} \rightarrow A_{0} \rightarrow 0$ is a semiabelian variety over $K_{0}$. Furthermore, if $A$ is of the form $\left(A_{0}\right)_{K_{1}}$ for some $A_{0}$ over $K_{0}$, we can choose $h$ to be the identity.

Proposition 2.7. Assume that $\operatorname{char}(K)=p$. Let $G$ be a semiabelian variety over $K$, such that $G$ descends to $K^{p^{n}}$ for all $n \geqslant 0$. Then $G$ descends to $K^{p^{\infty}}$.

Proof. Let $A$ be the abelian part of $G$, and let $T$ be its toric part. By Fact 2.6, $A$ descends to $K^{p^{n}}$ for all $n$. Using a suitable moduli space (namely the moduli space of abelian varieties equipped with a polarization of fixed degree and an $m$-level structure; see [27]), it follows that $A$ descends to $K^{p^{\infty}}$.

Now fix $A_{0}$ over $K^{p^{\infty}}$ such that $A \cong\left(A_{0}\right)_{K}$. It is classical that $\operatorname{Ext}(A, T) \simeq$ $\left(\operatorname{Ext}\left(A, \mathbb{G}_{m}\right)\right)^{t} \simeq(\hat{A})^{t}$, where $\hat{A}$ is the dual abelian variety of $A$, and is isomorphic to $\left(\hat{A}_{0}\right)_{K}$ (see, for example, [33]). Using Fact 2.6 again, and since $G$ descends to $K^{p^{n}}$ for each $n$, the isomorphism type of $G$ is parameterized by a point in $\hat{A}_{0}\left(\bigcap_{n} K^{p^{n}}\right)=\hat{A}_{0}\left(K^{p^{\infty}}\right)$; that is, $G$ descends to $K^{p^{\infty}}$.

Remark 2.8. Over a separably closed field $K$ of characteristic $p>0$, the semiabelian varieties over $K$ are exactly the commutative divisible algebraic groups over $K$. Indeed, let $H$ be commutative divisible, and consider the biggest connected affine subgroup of $H, T$. By divisibility it must be a torus, and, as $K$ is separably closed, it is defined over $K$ (and split over $K$ ), and $H / T$ is an abelian variety, by Chevalley's theorem ([30]).

2.2.1. Torsion. The behaviour of the torsion elements of $G$ is particularly important in characteristic $p$. The following classical facts will enable us to fix some notation for the rest of the paper.

Fact 2.9. Let $G$ be a semiabelian variety over $K$, written additively, and let

$$
0 \rightarrow T \rightarrow G \rightarrow A \rightarrow 0
$$

with $\operatorname{dim}(A)=a$ and $\operatorname{dim}(T)=t$

1. If $n$ is prime to $p=\operatorname{char}(K)$ or arbitrary in characteristic 0 , then $[n]: G \mapsto G$, $x \mapsto n x$ is a separable isogeny of degree (= separable degree) $n^{2 a+t}$. We denote by $G[n]$ the kernel of $[n]$, the points of $n$-torsion; then $G[n](\bar{K}) \cong(\mathbb{Z} / n \mathbb{Z})^{2 a+t}$. By separability, $G[n](\bar{K})=G[n](K)$.

From now on, $\operatorname{char}(K)=p>0$.

2. $[p]: G \mapsto G$ is an inseparable isogeny of degree $p^{2 a+t}$, and of inseparable degree at least $p^{a+t}$. Hence there is some $r, 0 \leqslant r \leqslant a$, such that, for every $n$,

$$
G\left[p^{n}\right](\bar{K})=\operatorname{Ker}\left[p^{n}\right](\bar{K}) \cong\left(\mathbb{Z} / p^{n} \mathbb{Z}\right)^{r} .
$$

We say that $G$ is ordinary if $r=a$ (note that tori are ordinary semiabelian varieties).

As $G\left[p^{n}\right](\bar{K})$ is finite, it is contained in $G(\bar{K})$, but not necessarily in $G(K)$. 
3. Let $G\left[p^{\infty}\right]$ or $G\left[p^{\infty}\right](\bar{K})$ denote the elements of $G$ with order a power of $p$, and let $G\left[p^{\prime}\right]$ or $G\left[p^{\prime}\right](\bar{K})$ denote the elements of $G$ with order prime to $p$. Then $G\left[p^{\prime}\right]=$ $G\left[p^{\prime}\right](K)$ is Zariski dense in $G$.

Note that, even for $G$ ordinary, we may well have that $G\left[p^{\infty}\right](K)=\{0\}$.

We will also need the following easy observations.

Fact 2.10. Let $0 \rightarrow G_{1} \rightarrow G_{2} \stackrel{f}{\rightarrow} G_{3} \rightarrow 0$ be an exact sequence of semiabelian varieties over $K$. Then for every $n$, the restriction of $f$ to $n$ torsion induces an exact sequence (in the category of groups), i.e.,

$$
0 \rightarrow G_{1}[n](\bar{K}) \rightarrow G_{2}[n](\bar{K}) \stackrel{f}{\rightarrow} G_{3}[n](\bar{K}) \rightarrow 0 .
$$

It follows in particular that, in all characteristics,

$$
0 \rightarrow \operatorname{Tor} G_{1} \rightarrow \operatorname{Tor} G_{2} \stackrel{f}{\rightarrow} \operatorname{Tor} G_{3} \rightarrow 0
$$

is an exact sequence of groups, where Tor $G$ denotes the group of all torsion elements of $G(\bar{K})$.

Divisibility by $p$ also behaves quite differently in $G(\bar{K})$ and in $G(K)$ when $\operatorname{char}(K)=p$. Let

$$
p^{\infty} G(K):=\bigcap_{n \geqslant 1}\left[p^{n}\right] G(K) .
$$

Proposition 2.11. $\quad$ 1. $G(K)$ is $n$-divisible for any $n$ prime to $p$.

2. For $n$ prime to $p$, for every $k, G[n](\bar{K})=G[n](K) \subset\left[p^{k}\right] G(K)$.

3. $G\left[p^{\prime}\right](\bar{K})=G\left[p^{\prime}\right](K)$ is a divisible subgroup of $G(K)$.

4. $p^{\infty} G(K)$ is n-divisible for any $n$ prime to $p$.

5. $p^{\infty} G(K)$ is infinite and Zariski dense in $G$.

6. $p^{\infty} G(K)$ is the biggest divisible subgroup of $G(K)$.

Proof. 1 to 5 are clear from previous facts.

6 follows from König's lemma and the finiteness of $G\left[p^{n}\right]$ for every $n$.

2.2.2. Isogenies and descent in char.p. We will not necessarily directly use all the classical facts about isogenies recalled below, but they give a picture of the various problems linked to descent questions in characteristic $p$. We will provide short elementary proofs when they exist.

In this section, $K$ is any separably closed field of characteristic $p>0$, and $G$ and $H$ are semiabelian varieties over $K$.

Note first that, if $G$ and $H$ are semiabelian varieties over $K$, and $f$ a morphism of algebraic groups $G_{L} \rightarrow H_{L}$ for some extension $L \supset K$, then $f$ is actually defined over $K$, i.e., $f=g_{L}$ for some $K$-morphism $g$ from $G$ to $H$ : by 2.4, the graph of $f$, which is a closed connected subgroup of $(G \times H)_{L}$, is also defined over $K$.

Recall that an isogeny is a surjective morphism of algebraic groups with finite kernel.

Let $G$ be a semiabelian variety over $K$. It is classical that for every $n \geqslant 1$ the relative $n$ th-Frobenius isogeny $F^{n}: G \longrightarrow G^{\left(p^{n}\right)}\left(G^{\left(p^{n}\right)}\right.$ descends to $\left.K^{p^{n}}\right)$ is purely inseparable 
of degree $p^{n \operatorname{dim} G}$, and admits a 'quasi-inverse' isogeny, the $n$ th-Verschiebung, denoted $V_{n}: G^{\left(p^{n}\right)} \longrightarrow G$, such that $V_{n} \circ F^{n}=\left[p^{n}\right]_{G}$ and $F^{n} \circ V_{n}=\left[p^{n}\right]_{G^{\left(p^{n}\right)}}$. It is easily seen, counting degrees, that the following holds.

Fact 2.12. If $G$ is ordinary, then, for every $n$, the Verschiebung $V_{n}$ is separable.

Lemma 2.13. Let $G$ be a semiabelian variety over $K$, and let $L$ be an extension of $K$. Then, if $a \in p^{n} G(L)$, there exists $b \in G(L)$ such that $a \in K\left(F^{n}(b)\right)$. So, if $G$ is over $K^{p^{n}}$, then $\left[p^{n}\right] G(K) \subset G\left(K^{p^{n}}\right)$, and in particular $p^{\infty} G(K)=p^{\infty} G\left(K^{p^{n}}\right)$.

Proof. Consider the $n$ th-Verschiebung $V_{n}$, described above. If $a \in p^{n} G(L)$, then $a=p^{n} b$ for some $b \in G(L)$, and $a=V_{n}\left(F^{n}(b)\right)$. If $G$ is over $K^{p^{n}}$, then the Verschiebung is also over $K^{p^{n}}$, and $a \in K^{p^{n}}\left(F^{n}(b)\right)=K^{p^{n}}$.

Abelian varieties have one specific very important property.

Fact 2.14. Let $A$ be an abelian variety over $K$. Then $A$ is isogenous over $K$ to a finite product of simple (i.e., which have no proper nontrivial closed connected subgroup) abelian varieties.

We will now recall some very classical facts about descent. We will try to point out where the difficulties arise, for our readers not completely familiar with the theory of abelian varieties in characteristic $p$.

In characteristic 0 , any semiabelian variety which is isogenous to one defined over some algebraically closed $K_{0}$ descends, in the sense above, to $K_{0}$ (i.e., Fact 2.15 applies). The situation is more complicated in characteristic $p$.

Fact 2.15. Let $K_{0} \subset K_{1}$, with $K_{0}$ algebraically closed. Let $G$ be a semiabelian variety over $K_{0}$, let $H$ be a semiabelian variety over $K_{1}$, and let $f$ be a separable isogeny from $G_{K_{1}}$ onto $H$. Then $H$ descends to $K_{0}$.

Proof. As $f$ is a separable isogeny, the kernel of $f$ is a finite closed subgroup of $G\left(K_{0}\right)$, $N$, of cardinality the degree (= separable degree) of $f$. Then $G^{\prime}:=G / N$ is a semiabelian variety over $K_{0}$, and $f$ induces an isomorphism from $H$ onto $G_{K_{1}}^{\prime}$.

The following is also classical, but more complicated and is only true for abelian varieties.

Fact 2.16. Let $K_{0} \subset K_{1}$, with $K_{0}$ algebraically closed. Let $A$ be an abelian variety over $K_{1}$, let $B$ be an abelian variety over $K_{0}$, and let $f$ be a separable isogeny from $A$ onto $B_{K_{1}}$. Then $A$ descends to $K_{0}$.

Proof. This is a particularly simple case of the 'proper base change theorem' (see, for example, in [15] or [24]).

Remark 2.17. Note that, in the case of dimension one, Fact 2.15 holds without the assumption that $f$ is separable. That follows easily from the fact that, in dimension one, an isogeny factors through some power of the Frobenius (see, for example, [36]).

We will give later (Remark 4.20) an example showing that Facts 2.15 and 2.16 do not hold without the separability assumption in dimension $>1$. 


\subsection{Relative Morley Rank}

In this section, $T$ will be a complete theory, and we work in a given $\kappa$-saturated model $M$, for $\kappa$ sufficiently big. We will here define the relative Morley rank, namely the Morley rank inside a given $\triangle$-definable set. This was called the internal Morley dimension in [16]. By an $M$-definable set (infinitely definable set) we mean a subset of some $M^{n}$ which is the intersection of a small $($ size $<\kappa)$ collection of definable subsets of $M^{n}$ (that is the set of realizations of a partial type over a small set of parameters). We will fix an $\mathbf{M}$-definable set $X \subseteq M^{n}$.

If $X$ is an infinitely definable subset of $M^{n}$, by a relatively definable subset of $X$ we mean a subset of the form $Z=X \cap Y$ for $Y \subseteq M^{n}$ definable with parameters. Then we define the Morley rank for relatively definable subsets $Z$ of $X$, as follows.

(i) $R M_{X}(Z) \geqslant 0$ if $Z$ is nonempty.

(ii) $R M_{X}(Z) \geqslant \alpha+1$ if there are $Z_{i} \subseteq Z$ for $i<\omega$ which are relatively definable subsets of $X$, such that $Z_{i} \cap Z_{j}=\emptyset$ for $i \neq j$ and $R M_{X}\left(Z_{i}\right) \geqslant \alpha$ for all $i$.

(iii) For limit ordinal $\alpha, R M_{X}(Z) \geqslant \alpha$ if $R M_{X}(Z) \geqslant \delta$ for all $\delta<\alpha$.

As in the absolute case, we obtain the (relative) Morley degree. Namely, suppose that $R M_{X}(Z)=\alpha<\infty$. Then there is a greatest positive natural number $d$ such that $Z$ can be partitioned into $d$ (relatively in $X$ ) definable sets $Z_{i}$ such that $R M_{X}\left(Z_{i}\right)=\alpha$ for all $i$.

We will say that $X$ has relative Morley rank if $R M_{X}(X)<\infty$.

Remark 2.18. (i) Suppose that $Y$ is a relatively definable subset of $X$. Then $R M_{X}(Y)=$ $R M_{Y}(Y)$.

(ii) We can also talk about the relative Morley $\operatorname{rank} R M_{X}(p)$ of a complete type $p$ of an element of $X$ over a set of parameters. It will just be the infimum of the relative Morley ranks of the (relatively) definable subsets of $X$ which are in $p$.

(iii) Suppose that $T$ is countable and that $X$ is $\mathbb{M}$-definable over a countable set of parameters $A_{0}$. Then $X$ has relative Morley rank if and only if for any countable set of parameters $A \supseteq A_{0}$ there are only countably many complete types over $A$ extending $X$.

Now suppose that $X, Y$ are $M$-definable sets and that $f: X \rightarrow Y$ is a surjective definable function. By definability of $f$ we mean that $f$ is the restriction to $X$ of some definable function on a definable superset of $X$. Note that then each fibre $f^{-1}(c)$ of $f$ is a relatively definable subset of $X$, so we can talk about its relative Morley rank (with respect to $X$ or to itself, which will be the same by Remark 2.18(i)).

Lemma 2.19. Suppose that $X, Y$ are $\mathrm{M}$-definable sets and that $f: X \rightarrow Y$ is surjective and definable.

(i) Suppose that $R M_{Y}(Y)=\beta$ and that, for each $c \in Y, R M_{X}\left(f^{-1}(c)\right) \leqslant \alpha$. Then $R M_{X}(X) \leqslant \alpha(\beta+1)$ if $\alpha>0$, and $\leqslant \beta$ if $\alpha=0$.

(ii) $R M_{Y}(Y) \leqslant R M_{X}(X)$. 
Proof. (i) This is proved in the definable (absolute) case by Shelah [35] (Chapter V, Theorem 7.8) and Erimbetov [14]. Martin Ziegler [40] also gives a self-contained proof. We point out briefly how Ziegler's proof (see $\S 2$ of [40]) adapts to the more general context.

Case 1, when $\alpha=0$, [40] works word-for-word.

Case 2, when $\alpha>0$. Work by induction on $\beta$. We may assume that $Y$ has 'relative Morley degree' 1 (with respect to itself). Suppose for a contradiction that $\alpha \beta+\alpha<R M_{X}(X)$. Lemma 3 of [40] applies, yielding a relatively definable subset $X^{\prime}$ of $X$, such that $\alpha \beta<$ $R M_{X}\left(X^{\prime}\right)$ and such that the 'generic fibre' of $f \mid X^{\prime}$ has finitely many, say $k$, elements (where possibly $k=0$ ). We now apply compactness to find a relatively definable subset $Y^{*}$ of $Y$ such that, for all $b \in Y^{*}, f^{-1}(b) \cap X^{\prime}$ has at most $k$ elements. Let $Y^{\prime}=Y \backslash Y^{*}$ be a relatively definable subset of $Y$ such that $R M_{Y}\left(Y^{\prime}\right)=\beta^{\prime}<\beta$. By Case $1, X^{\prime} \cap f^{-1}\left(Y^{*}\right)$ has relative Morley rank $\leqslant \beta$, whereby the relative Morley rank of $X^{\prime \prime}=X^{\prime} \cap f^{-1}\left(Y^{\prime}\right)$ is $>\alpha \beta \geqslant \alpha\left(\beta^{\prime}+1\right)$. This contradicts the induction hypothesis applied to $f \mid X^{\prime \prime}: X^{\prime \prime} \rightarrow Y^{\prime}$.

(ii) is easier, and has the same inductive proof as in the definable (absolute) case, bearing in mind that, because $f$ is the restriction to $X$ of a definable function on a definable superset of $X$, the preimage under $f$ of any relatively definable subset of $Y$ is a relatively definable subset of $X$.

If $X=G$ is an $M$-definable group with relative Morley rank, then some of the general theory of totally transcendental groups applies (as already mentioned inside Definition 4.0 of [16]). For example, we have the DCC (descending chain condition) on relatively definable subgroups, yielding that $G$ is connected-by-finite among other things. And this is really all we will be using about groups of finite relative Morley rank.

We now consider an exact sequence of M-definable groups $1 \rightarrow G_{1} \stackrel{h}{\rightarrow} G_{2} \rightarrow G_{3} \rightarrow 1$. We can assume that $G_{1}=\operatorname{Ker}(h) \subseteq G_{2}$, as the relative Morley rank is preserved by definable bijection, and note again that $G_{1}$ is then a relatively definable (normal) subgroup of $G_{2}$. With this notation we have the following corollary, which follows immediately from Lemma 2.19:

Corollary 2.20. Suppose that $G_{1}$ and $G_{3}$ have (finite) relative Morley rank. Then so does $G_{2}$.

We complete this section with some additional comments and examples. First, we obtain the usual (absolute) Morley rank of a definable set $Z \subseteq M^{n}$ by taking $X$ to be $M^{n}$ in the definition at the beginning of this subsection. Of course the Morley rank can be defined directly for complete types (over a saturated enough model $M$ ), by $R M(p(x))=\alpha$ if $p(x)$ is isolated in the subspace of $S_{x}(M)$ obtained by removing the set of types of Morley rank $<\alpha$. Here, the ambient space of types is $S_{x}(M)$. We can make the analogous definition for relative Morley rank $R M_{X}(p)$, by working in the space $S_{X}(M)$ of complete types over $M$ extending the type-definable set $X$. In any case it should be clear to the reader that $R M_{X}(p)$ need not coincide with $R M(p)$. For example, suppose that $X$ is a so-called minimal type-definable set: namely $X$ is infinite and every relatively definable subset of $X$ is finite or cofinite (in $X$ ). Then there is a unique nonalgebraic complete type 
over $M$ extending $X$, say $p(x)$. Moreover, $R M_{X}(p)=1$. But $R M(p)$ may be undefined (i.e., $\infty)$. This is precisely the case when $M=K$ is a separably closed, nonalgebraically closed field, and $X=k=\bigcap_{n} K^{p^{n}} . X$ is type definable and minimal. For $p(x) \in S_{x}(M)$ the 'generic' type of $X$ as above, $R M_{X}(p)=1$, but $R M(p)=\infty$, because otherwise there would be a formula in $p$ of ordinal valued Morley rank, and there are no such (nonalgebraic) formulas in the theory of separably closed fields.

\section{The $\sharp$ functor}

Here, $K$ will be either a separably closed field of characteristic $p>0$ and finite degree of imperfection, or a differentially closed field of characteristic 0 (so with distinguished derivation $\partial$ ). We distinguish the cases by 'characteristic $p$ ', 'characteristic 0 '. In the characteristic $p$ case, we will take $K$ to be say $\omega_{1}$-saturated, so as to be able to do model theory, although this will not always be necessary. Definability will mean in the sense of the structure $K$. In the characteristic 0 case, as $\mathrm{DCF}_{0}$ is $\omega$-stable, we have $D C C$ on definable subgroups of a definable group, so any $\wedge$-definable group is definable. In the characteristic $p$ case, by stability, any $\mathbb{M}$-definable subgroup is an intersection of at most countably many definable groups.

Definition 3.1. Let $G$ be a semiabelian variety over $K$. Then $G^{\sharp}$ is the smallest M-definable subgroup of $G(K)$ which is Zariski dense in $G$.

Various equivalent characterizations of $p^{\infty} G(K)$ were given in [8]. In particular, it was shown ([8], Proposition 3.6) that $p^{\infty} G(K)$ is the unique divisible subgroup of $G(K)$ which is Zariski dense in $G$. But the following one was omitted at the time.

Proposition 3.2. Suppose that $\operatorname{char}(K)=p$, and let $G$ be a semiabelian variety over $K$. Then $p^{\infty} G(K)$ is the smallest $\mathrm{M}$-definable group of $G(K)$ which is Zariski dense in $G$; hence $p^{\infty} G(K)=G^{\sharp}$.

Proof. Let $H$ be any $₫$-definable subgroup of $G(K)$, also Zariski dense in $G$. By stability, $H$ is a decreasing intersection of definable subgroups of $G(K),\left(H_{i}\right)_{i \in I}$. Certainly each $H_{i}$ is itself Zariski dense in $G$. By [7] Corollary 4.16, the connected component of $H_{i}, C_{i}$, is also definable in $G(K)$ and has finite index in $H_{i}$. It follows that it is also Zariski dense in $G$.

Now, for every $r \geqslant 1$ the (definable) subgroup $\left[p^{r}\right] C_{i}$ is also Zariski dense in $G$. It follows, by compactness and saturation, that $\bigcap_{n \geqslant 1}\left[p^{n}\right] C_{i}$ is also Zariski dense in $G$. But $\bigcap_{n \geqslant 1}\left[p^{n}\right] C_{i}$ is a divisible group, and by the remark above, $p^{\infty} G(K)=\bigcap_{n \geqslant 1}\left[p^{n}\right] C_{i}$ for every $i$, and is hence contained in $H$.

In characteristic $0, G^{\sharp}$ is sometimes called the 'Manin kernel' (see [20]). Alternative characterizations and key properties in arbitrary characteristic are given in the following lemma.

Lemma 3.3. (i) $G^{\sharp}$ can also be characterized as the smallest $\mathrm{M}$-definable subgroup of $G(K)$ which contains the (prime-to-p, in the char. $p$ case) torsion of $G$. 
(ii) $G^{\sharp}$ is connected (no relatively definable subgroup of finite index), and of finite $U$-rank in char. $p$, and finite Morley rank in char. 0 .

(iii) If $G=\left(G_{0}\right)_{K}$ for some $G_{0}$ over the constants $\mathcal{C}$ of $K$, then $G^{\sharp}=G(\mathcal{C})$.

Proof. (i) Recall first that the (prime-to- $p$ ) torsion is contained in $G(K)$. In the characteristic $p$ case, $G^{\sharp}=p^{\infty} G(K)$ does contain the prime-to- $p$ torsion. On the other hand, as the prime-to- $p$ torsion is Zariski dense in $G$, any subgroup of $G$ containing the prime-to- $p$ torsion is Zariski dense. So the lemma is established in characteristic $p$. The characteristic 0 case is well known and due originally to Buium. See, for example, Lemma 4.2 of [28] where it is proved that any definable Zariski-dense subgroup of a connected commutative algebraic group $G$ contains $\operatorname{Tor}(G)$.

(ii) $G^{\sharp}$ is connected as any finite index subgroup of a Zariski-dense subgroup is also Zariski dense. In the characteristic 0 case, Buium [10] showed that $G^{\sharp}$ has finite Morley rank. An account, using D-groups, appears in [6]. In the characteristic $p$ case, finite $U$-rank of $G^{\sharp}$ was first shown by Hrushovski in [16], and can also be seen to follow easily from Lemma 2.13.

(iii) In characteristic $p$, this is a direct consequence of Lemma 2.13 or of the fact that $G(\mathcal{C})$ is both divisible and Zariski dense in $G$. In characteristic 0, it can be seen as follows. Assume $G$ to be defined over $\mathcal{C}$. Note that $G(\mathcal{C})$ is definable in the differentially closed field $K$. As $\mathcal{C}$ is algebraically closed, $G(\mathcal{C})$ is Zariski dense in $G$; hence $G^{\sharp} \subseteq G(\mathcal{C})$. If $G^{\sharp} \subsetneq G(\mathcal{C}), G^{\sharp}=H(\mathcal{C})$ for some proper algebraic subgroup $H$ of $G$ over $\mathcal{C}$, and then $H(\mathcal{C})$ could not be Zariski dense in $G$.

Lemma 3.4. Let $G, H$ be semiabelian varieties over $K$, and let $f: G \rightarrow H$ be a (not necessarily separable) rational $K$-homomorphism. Then the following hold.

(i) $f\left(G^{\sharp}\right) \subseteq H^{\sharp}$.

(ii) If $f$ is dominant, then $f\left(G^{\sharp}\right)=H^{\sharp}$.

Proof. (i) Let $\operatorname{Tor}_{p^{\prime}}(G)$ be the prime-to- $p$ torsion (so all the torsion in char.0). Note that $f\left(\operatorname{Tor}_{p^{\prime}}(G)\right) \subseteq \operatorname{Tor}_{p^{\prime}}(H)$. If (i) fails, then $D=f\left(G^{\sharp}\right) \cap H^{\sharp}$ is a proper M-definable subgroup of $H(K)$ which by Lemma 3.3 contains $f\left(\operatorname{Tor}_{p^{\prime}}(G)\right)$. But then $f^{-1}(D) \cap G(K)$ is an \-definable subgroup of $G(K)$ which contains $\operatorname{Tor}_{p^{\prime}}(G)$ and is properly contained in $G^{\sharp}$, contradicting Lemma 3.3.

(ii) Note that $f\left(G^{\sharp}\right)$ is $M$-definable (by $\omega_{1}$-saturation in characteristic $p$, since in this case $\left.f\left(\cap G_{i}\right)=\cap f\left(G_{i}\right)\right)$, and, since $f$ is dominant, $f\left(G^{\sharp}\right)$ must be Zariski dense in $H$. By part (i), and the definition of $H^{\sharp}, f\left(G^{\sharp}\right)=H^{\sharp}$.

Remark 3.5 (Characteristic $p$ ). Let $f: G \rightarrow H$ be as in the hypothesis of Lemma 3.4(ii). If $f$ is separable (that is, induces a separable extension of function fields) then, as we remarked in Fact 2.3, $f_{\mid G(K)}: G(K) \rightarrow H(K)$ is surjective. If $f$ is not separable, $f$ may no longer be surjective at the level of $K$-rational points, but nevertheless Lemma 3.4(ii) says it is surjective on the $\sharp$-points when $K$ is $\omega_{1}$-saturated. 
Note however that, if $f$ is an isogeny, $f\left(p^{\infty} G(K)\right)=p^{\infty} H(K)$ without any saturation assumption (if $f$ has degree of inseparability $n$, then $\left[p^{n}\right] H(K) \subseteq f(G(K)$ ), and one concludes by König's lemma).

By Lemma 3.4(i), we can consider $\sharp$ as a functor from the category of semiabelian varieties over $K$ to the category of $\bigwedge$-definable groups in $K$. It is natural to ask whether $\sharp$ preserves exact sequences, and this is an important theme of the paper.

Recall that, by an exact sequence of algebraic groups defined over $K$, we mean that the homomorphisms are not only over $K$ but also separable. Consider two semiabelian varieties $G_{2}, G_{3}$ over $K$, a separable surjective $K$-homomorphism $f: G_{2} \rightarrow G_{3}$, with $\operatorname{Ker}(f)=G_{1}$ connected and thus a semiabelian subvariety of $G_{2}$ over $K$. Then, by Fact 2.3, the sequence $0 \rightarrow G_{1}(K) \rightarrow G_{2}(K) \rightarrow G_{3}(K) \rightarrow 0$ clearly remains exact (in the category of definable groups in $K)$. By Lemma 3.4, the sequence

$$
0 \rightarrow G_{1}^{\sharp} \rightarrow G_{2}^{\sharp} \rightarrow G_{3}^{\sharp} \rightarrow 0
$$

will be exact if and only if

$$
G_{1}^{\sharp}=G_{1}(K) \cap G_{2}^{\sharp} .
$$

So the group $\left(G_{1}(K) \cap G_{2}^{\sharp}\right) / G_{1}^{\sharp}$ is the obstruction to exactness.

In the characteristic 0 case, this group, which is clearly of finite Morley rank, can be seen to be connected and embeddable in a vector group. By Lemma 4.2 of [28], for example, $G_{1}(K) / G_{1}^{\sharp}$ (as a group definable in $K$ by elimination of imaginaries) embeds definably in $(K,+)^{n}$ for some $n$. Hence $\left(G_{1}(K) \cap G_{2}^{\sharp}\right) / G_{1}^{\sharp}$ also embeds in $(K,+)^{n}$, and as such is a (finite-dimensional) vector space over the field of constants of $K$. Hence $\left(G_{1}(K) \cap G_{2}^{\sharp}\right) / G_{1}^{\sharp}$ is connected. Note that, as $G_{1}^{\sharp}$ is also connected, it follows that $G_{1}(K) \cap G_{2}^{\sharp}$ itself is also connected.

The characteristic $p$ case is different in an interesting way. Note, first, that the group $\left(G_{1}(K) \cap G_{2}^{\sharp}\right) / G_{1}^{\sharp}$ is not even infinitely definable; it is the quotient of two $\wedge$-definable groups. Such groups are usually called 'hyperdefinable'.

We will recall the (model-theoretic) definition of a connected component. First, if $G$ is an $\aleph$-definable group in a stable theory, then we have $D C C$ on intersections of uniformly relatively definable subgroups (see [29] or [39]). What this means is that, if $\phi(x, y)$ is a formula, then the intersection of any collection of subgroups of $G$ relatively defined by some instance of $\phi(x, y)$ is a finite subintersection. It follows that, working in a saturated model, say, the intersection of all relatively definable subgroups of $G$ of finite index is the intersection of at most $|L|$ many (where $L$ is the language). We call this intersection, $G^{0}$, the connected component of $G$. It is normal, and type definable over the same set of parameters that $G$ is. Moreover, $G / G^{0}$ is naturally a profinite group. In the $\omega$-stable case (or the relative finite Morley rank case as in $\S 2.3$ ), by $D C C$ on relatively definable subgroups, $G^{0}$ will itself be relatively definable and of finite index in $G$.

Lemma 3.6 (Characteristic $p$ ). Let $G_{1}$ be a semiabelian subvariety of the semiabelian variety $G_{2}$, both defined over $K$. Then $G_{1}^{\sharp}$ is the connected component of $G_{1}(K) \cap G_{2}^{\sharp}$. 
Proof. First, by $3.4, G_{1}^{\sharp}$ is a subgroup of $G_{1}(K) \cap G_{2}^{\sharp}$. By Lemma $3.3, G_{1}(K) \cap G_{2}^{\sharp}$ is \-definable of finite $U$-rank. Hence, for any $H$ \-definable subgroup of $G_{1}(K) \cap G_{2}^{\sharp}$, classical $U$-rank inequalities for groups give us that $U(H[n])+U([n] H)=U(H)$. As for each $n$ the $n$-torsion of $H$ is finite, $U([n] H)=U(H)$. It follows that $H$ is connected if and only if it is divisible: if $H$ is connected, then any proper infinitely definable subgroup of $H$ has strictly smaller $U$-rank than $H$, so, for every $n,[n] H=H$, and $H$ is divisible. But $G_{1}^{\sharp}$ is the biggest divisible subgroup of $G_{1}(K)$. Thus $G_{1}^{\sharp}$ must coincide with the connected component of $G_{1}(K) \cap G_{2}^{\sharp}$.

Remark 3.7. By Lemma 3.6, the quotient $\left(G_{1}(K) \cap G_{2}^{\sharp}\right) / G_{1}^{\sharp}$ is a profinite group. If $G_{2}^{\sharp}$ had relative Morley rank, the quotient would have to be finite (as remarked above). We will see in $\S 4$ an example where the quotient is infinite, and give an explicit description of this quotient in terms of suitable Tate modules.

For the record, we now mention cases (in characteristic $p$ ) where $G^{\sharp}$ has (finite) relative Morley rank.

Fact 3.8 (Characteristic $p$ ). Let $G$ be a semiabelian variety over $K$. Then the following hold.

(i) If $G$ descends to $K^{p^{\infty}}$ (in particular, if $G$ is an algebraic torus), then $G^{\sharp}$ has finite relative Morley rank.

(ii) If $G=A$ is an abelian variety, then $A^{\sharp}$ has finite relative Morley rank.

Proof. (i) We may assume that $G=\left(G_{0}\right)_{K}$ for some $G_{0}$ over $K^{p^{\infty}}$. Then, by Lemma 2.13, $G^{\sharp}=p^{\infty} G(K)=G\left(K^{p^{\infty}}\right)$. As $K^{p^{\infty}}$ is a 'pure' algebraically closed field inside $K, G\left(K^{p^{\infty}}\right)$ has relative Morley rank equal to the (algebraic) dimension of $G$.

(ii) The abelian variety $A$ is isogenous to a product of simple abelian varieties. So we may reduce to the case where $A$ is simple. In that case, $A^{\sharp}$ has no proper infinite definable subgroup (Lemma 2.16 in [16] or Corollary 3.8 in [8]). By stability, $A^{\sharp}$ has no proper infinite $\wedge$-definable subgroup. We will now use an appropriate version of Zilber's indecomposability theorem to see that $A^{\sharp}$ has finite relative Morley rank. As $A^{\sharp}$ has finite $U$-rank, there is some small submodel $K_{0}$ (over which $A^{\sharp}$ is defined) and a complete type $p(x)$ over $K_{0}$ extending ' $x \in A^{\sharp}$ ', which has $U$-rank 1 (and is of course stationary). Let $Y \subseteq A^{\sharp}$ be the set of realizations of $p$. Then $Y$ is an $M$-definable subset of $A^{\sharp}$ which is 'minimal', namely $Y$ is infinite, and every relatively definable subset of $Y$ is either finite or cofinite. We claim that $Y$ is 'indecomposable' in $A^{\sharp}$, namely, for each relatively definable subgroup $H$ of $A^{\sharp},|Y / H|$ is 1 or infinite. For, if not, then, as remarked earlier, the intersection of all the images of $H$ under automorphisms fixing $K_{0}$ pointwise will be a finite subintersection $H_{0}$, now defined over $K_{0}$, and we will have $\left|Y / H_{0}\right|>1$ and finite, contradicting the stationarity (or even completeness) of $p$. Let now $X$ be a translate of $Y$ which contains the identity 0 . Then $X$ is still a minimal $M$-definable subset of $A^{\sharp}$. Moreover, Theorem 3.6.11 of [39] or Theorem 6.10 of [29] applies to this situation, to yield that the subgroup $B$ say of $A^{\sharp}$ which is generated by $X$ is $M$-definable and moreover of 
the form $X+X+\cdots+X$ ( $m$ times) for some $m$. As noted above, it follows that $B=A^{\sharp}$, and so the function $f: X^{m} \rightarrow A^{\sharp}$ is a definable surjective function between $M$-definable sets, in the sense of $\S 2.3$. But, as $X$ is minimal, clearly $R M_{X}(X)=1$ and $R M_{X^{m}}\left(X^{m}\right)=m$. By Lemma 2.19(ii), $A^{\sharp}$ has finite relative Morley rank too.

Let us remark that, in the context of the proof of (ii) above, when $A$ is a simple abelian variety over $K$ which does not descend to the constants, then, via the dichotomy theorem for minimal types in separably closed fields, $A^{\sharp}$ is connected, of $U$-rank 1 , and hence has relative Morley rank 1 . However, we wanted to avoid the appeal to the dichotomy theorem, and hence above we use the proof involving a version of Zilber's indecomposability theorem.

\section{Characteristic $p$}

Here, we follow the 'naive' algebraic approach which works in a very simple way in characteristic $p$. In order to highlight the uniformity with char. 0 , we will, in the next section, adopt a point of view closer to algebraic geometry.

We deal now with the characteristic $p$ case. Let $G$ be a semiabelian variety over any model $(K, \partial)$ of $\mathrm{CHF}_{p, 1}$, that is, any separably closed field of degree of imperfection 1 .

\subsection{Torsion points, Tate modules, and descent}

We make no saturation hypothesis for the moment.

Definition 4.1. We define $\tilde{G}$ as the inverse limit

$$
\tilde{G}:=\lim _{\leftarrow}(G \stackrel{[p]}{\longleftarrow} G \stackrel{[p]}{\longleftarrow} \cdots) .
$$

In particular, for $L$ an extension of $K$ (we will mainly consider $L=K$ or $L=\bar{K}$ ),

$$
\tilde{G}(L)=\left\{\left(x_{i}\right)_{i \in \mathbb{N}} \in G(L)^{\mathbb{N}}: \forall i \geqslant 0, x_{i}=[p] x_{i+1}\right\} .
$$

Let $\pi_{G}$ be the projection onto the 'left component' $G(L)$. The kernel of $\pi_{G}$ is precisely $T_{p} G(L)$, where $T_{p} G$ is the Tate module of $G$.

Its $L$-points in an arbitrary algebraically closed extension $L$ of $K$ coincide with the sequences of torsion points in $\bar{K}$,

$$
T_{p} G(\bar{K})=\left\{\left(x_{i}\right)_{i \in \mathbb{N}} \in G(\bar{K})^{\mathbb{N}}: x_{0}=0, \forall i \geqslant 0, x_{i}=[p] x_{i+1}\right\} .
$$

By definition, $\tilde{G}$ is a proalgebraic group, i.e., an inverse limit of algebraic groups. In $\S 5.2, \tilde{G}$ will be viewed as a group scheme. Here, we adopt a more naive point of view, closer to model theory. Objects such as $\tilde{G}(K)$ and $T_{p} G(K)$ are what are called '*-definable' groups in $K$ (projective limits of definable groups).

Let us note that, for a given $g_{0} \in G(K), g_{0} \in G^{\sharp}$ if and only if there is some $\left(x_{i}\right)_{i \in \mathbb{N}} \in$ $\tilde{G}(K)$ with $g_{0}=x_{0}$; we deduce directly from this the relation between the Tate module of $G$ and $G^{\sharp}$.

Lemma 4.2. The morphism $\pi_{G}$ induces an exact sequence of $*$-definable groups.

$$
0 \rightarrow T_{p} G(K) \rightarrow \tilde{G}(K) \stackrel{\pi_{G}}{\rightarrow} G^{\sharp} \rightarrow 0 .
$$


In the case of ordinary semiabelian varieties, if the dimension of the abelian part is $a, T_{p} G(\bar{K}) \simeq \mathbb{Z}_{p}^{a}$ (see Fact 2.9). We relate now the part of the $p^{\infty}$-torsion lying in $K$ with issues of descent. Most of the following results are certainly well known; see, for example, [38] for the description of the torsion of $G$ for abelian schemes of maximal Kodaira-Spencer rank. But we have found no systematic exposition which we could quote, and, furthermore, we choose to give here very elementary proofs which are suitable for our purpose.

Proposition 4.3. Let $G$ be an ordinary semiabelian variety over $K$. Then, for every $n$, $G\left[p^{n}\right](\bar{K})=G\left[p^{n}\right](K)$ if and only if $G$ descends to $K^{p^{n}}$. In particular, $G$ descends to $K^{p^{\infty}}$ if and only if $G\left[p^{\infty}\right](\bar{K})=G\left[p^{\infty}\right](K)$ if and only if $T_{p} G(\bar{K})=T_{p} G(K)$.

Proof. Let us fix $n \geqslant 1$. If $G$ descends to $K^{p^{n}}$, we may assume that $G=\left(G_{0}\right)_{K}$ for some $G_{0}$ over $K^{p^{n}}$. Since $G_{0}$ is ordinary, $V_{n}$ is separable, and the geometric points of the kernel of $V_{n}$ are $K^{p^{n}}$-rational, and, since $\left[p^{n}\right]=V_{n} \circ F^{n}, G_{0}\left[p^{n}\right](\bar{K})=F^{-n}\left(\operatorname{Ker}\left(V_{n}\right)(\bar{K})\right) \subseteq$ $G_{0}(K)$.

Conversely, assume that $G\left[p^{n}\right](\bar{K}) \subseteq G(K)$. Since $V_{n}$ is separable, $G$ is isomorphic to the quotient $F^{n} G / \operatorname{Ker}\left(V_{n}\right)$. But $\operatorname{Ker}\left(V_{n}\right)(\bar{K})=F^{n}\left(G\left[p^{n}\right](\bar{K})\right)$ is a finite group of $K^{p^{n}}$-rational points; hence $F^{n} G / \operatorname{Ker}\left(V_{n}\right)$ descends to $K^{p^{n}}$. The 'in particular' statement follows from Proposition 2.7.

Corollary 4.4. Let $K_{0}$ be an algebraically closed field, and let $K_{1}>K_{0}$ be a finitely generated extension of $K_{0}$. Let $G$ be an ordinary semiabelian variety over $K_{1}$. If $G\left[p^{\infty}\right]\left(\overline{K_{1}}\right)=G\left[p^{\infty}\right]\left(K_{1}\right)$, then $G$ descends to $K_{0}$.

Proof. As $K_{0}$ is algebraically closed, $K_{1}$ is a separable extension of $K_{0}$, and hence it is contained in the separable closure of $K_{0}\left(t_{1}, \ldots, t_{n}\right)$ for $t_{1}, \ldots, t_{n}$ algebraically independent. Then (Fact 2.2) there is a separably closed field $K$ of degree of imperfection 1, extending $K_{1}$, and such that $K_{0}=K^{p^{\infty}}$. We can now apply Proposition 4.3 to conclude that $G$ descends to $K^{p^{\infty}}$.

This yields easily the following result (compare with Fact 2.15; here $f$ is no longer separable but $G$ is ordinary).

Corollary 4.5. Let $G$ be an ordinary semiabelian variety over some algebraically closed field $K_{0}$. If $H$ is any semiabelian variety over $K_{1}>K_{0}$ such that there is an isogeny $f$ from $G_{K_{1}}$ onto $H$, then $H$ descends to $K_{0}$.

Proof. Let $K_{2}<K_{1}$ be a finitely generated extension of $K_{0}$ over which $H$ and the isogeny $f$ from $G$ to $H$ are defined. We claim first that any point of $p^{\infty}$-torsion in $H$ is the image of a point of $p^{\infty}$-torsion in $G$ : indeed, let $h \in H\left[p^{\infty}\right]\left(\overline{K_{2}}\right)$, i.e., for some $m,\left[p^{m}\right] h=0$. Let $g \in G\left(\overline{K_{2}}\right)$ be a preimage of $h, f(g)=h$. Then $\left[p^{m}\right] g \in \operatorname{Ker} f$. Let $n \geqslant 1$ be the order of the finite group $(\operatorname{Ker} f)\left(\overline{K_{2}}\right)$. Then $n=p^{r} d$, where $d$ is prime to $p$. Write $1=u d+v p^{m}$, $u, v \in \mathbb{Z}$. Then $g=[u d] g+\left[v p^{m}\right] g$, so $h=f(g)=f([u d] g)$, with $\left[p^{r+m}\right][u d] g=0$.

Now, as $K_{0}$ is algebraically closed, $G\left[p^{\infty}\right]\left(\overline{K_{2}}\right)=G\left[p^{\infty}\right]\left(K_{0}\right)$, and hence by the above claim $H\left[p^{\infty}\right]\left(\overline{K_{2}}\right) \subseteq f\left(G\left[p^{\infty}\right]\left(\overline{K_{2}}\right)\right)=f\left(G\left[p^{\infty}\right]\left(K_{0}\right)\right) \subseteq H\left[p^{\infty}\right]\left(K_{2}\right)$. We can now apply Corollary 4.4 . 
Corollary 4.6. Let $K_{0}<K_{1}$ be an extension of fields with $K_{0}$ algebraically closed, and let $0 \longrightarrow C \longrightarrow B \longrightarrow A \longrightarrow 0$ be an exact sequence of ordinary abelian varieties over $K_{1}$, such that $A$ and $C$ descend to $K_{0}$. Then $B$ descends to $K_{0}$.

Proof. By Poincaré's reducibility theorem, $B$ is isogenous to $A \times C$, which descends to $K_{0}$, and we just have to apply Corollary 4.5 .

Remark 4.7. See Remark 4.20 for a counterexample if one does not require the abelian varieties to be ordinary.

We complete this section with some basic remarks about torsion in $G(K) / G^{\sharp}$ $\left(=G(K) / p^{\infty} G(K)\right)$ in characteristic $p$ which will immediately enable us to describe the link between the question of relative Morley rank and that of preservation of exactness.

Lemma 4.8. Let $G$ be a semiabelian variety over $K$.

(i) $G\left[p^{\infty}\right](K)$ is a direct sum of a divisible group and a finite group.

(ii) $G(K) / p^{\infty} G(K)$ has finite torsion.

(iii) If $G$ descends to $K^{p^{\infty}}$, then $G(K) / p^{\infty} G(K)$ is torsion free.

(iv) If $G(K)$ has trivial $p$-torsion then $G(K) / p^{\infty} G(K)$ is torsion free.

Proof. (i) $G\left[p^{\infty}\right](K)$ is a subgroup of $G\left[p^{\infty}\right](\bar{K})$ which is a finite direct sum of copies of the Prüfer group $\mathbb{Z}_{p}$.

As $p^{\infty} G(K)$ is divisible, if $g \in G(K)$ and $n g \in p^{\infty} G(K)$, then there is $h \in p^{\infty} G(K)$ such that $n g=n h$, whereby $n(g-h)=0$; so $g$ is congruent $\bmod p^{\infty} G(K)$ to an element of order $n$. We know that $p^{\infty} G(K)$ contains all the prime-to- $p$-torsion of $G$. On the other hand, by (i), $G\left[p^{\infty}\right](K) / p^{\infty} G(K)$ is finite. This gives (ii) immediately.

Similarly, for cases (iii) and (iv), where $p^{\infty} G(K)$ contains all the torsion of $G(K)$.

Proposition 4.9. Suppose that $K$ is $\omega_{1}$-saturated, and let $G$ be a semiabelian variety over $K, 0 \rightarrow T \rightarrow G \rightarrow A \rightarrow 0$. Then the following are equivalent.

(i) $G^{\sharp}$ has relative Morley rank.

(ii) The sequence $0 \rightarrow T^{\sharp} \rightarrow G^{\sharp} \rightarrow A^{\sharp} \rightarrow 0$ is exact.

(iii) $\left(T(K) \cap G^{\sharp}\right) / T^{\sharp}$ is finite.

(iv) $T(K) \cap G^{\sharp}$ is divisible.

Proof. By the previous lemma, as $T$ has no $p$-torsion, $T(K) / T^{\sharp}$ is torsion free. Also note that $T^{\sharp}=T(\mathcal{C})$ is divisible and is the connected component of $T(K) \cap G^{\sharp}(3.6)$. Hence $\left(T(K) \cap G^{\sharp}\right) / T^{\sharp}$ is finite if and only if it is trivial if and only if the sequence $0 \rightarrow T^{\sharp} \rightarrow G^{\sharp} \rightarrow A^{\sharp} \rightarrow 0$ is exact. And, moreover, these conditions are equivalent to the divisibility of $T(K) \cap G^{\sharp}$. This gives the equivalence of (ii), (iii), and (iv).

On the other hand, if $G^{\sharp}$ has finite relative Morley rank, then every relatively definable subgroup is connected by finite, so (i) implies (iii). Conversely, we have seen (3.8) that both $T^{\sharp}$ and $A^{\sharp}$ have relative Morley rank. By 2.20, the exactness of the sequence implies that $G^{\sharp}$ also has relative Morley rank. Thus (ii) implies (i). 


\subsection{Exactness and descent}

We now assume that $K$ is $\omega_{1}$-saturated.

Proposition 4.10. Let $0 \longrightarrow G_{1} \stackrel{h}{\longrightarrow} G_{2} \stackrel{f}{\longrightarrow} G_{3} \longrightarrow 0$ be an exact sequence of semiabelian varieties over $K$. Let $f^{\omega}$ be the induced morphism of $\mathbb{Z}_{p}$-modules from $T_{p} G_{2}(K)$ to $T_{p} G_{3}(K)$. Then there is an isomorphism

$$
\phi:\left(h\left(G_{1}(K)\right) \cap G_{2}^{\sharp}\right) / h\left(G_{1}^{\sharp}\right) \stackrel{\longrightarrow}{\longrightarrow} T_{p} G_{3}(K) / f^{\omega}\left(T_{p} G_{2}(K)\right) .
$$

Proof. Note that we may assume that $h$ is the inclusion. We first define $\phi$. Let $g$ be in $G_{1}(K) \cap p^{\infty} G_{2}(K)$. There exists an element $\left(g_{i}\right)_{i \in \mathbb{N}}$ in $T_{p}\left(G_{2}(K), g\right)$ (the fibre of $\tilde{G}_{2}(K)$ over $\left.g\right)$, with $g_{0}=g \in G_{1}(K)$. Hence $f^{\omega}\left(\left(g_{i}\right)\right) \in T_{p} G_{3}(K)$. We check that it gives a well-defined map (even a group homomorphism) from $G_{1}(K) \cap p^{\infty} G_{2}(K)$ to $T_{p} G_{3}(K) / f^{\omega}\left(T_{p} G_{2}(K)\right)$ : if $\left(g_{i}^{\prime}\right)_{i \in N}$ is another element in $T_{p}\left(G_{2}(K), g\right)$, then $\left(g_{i}\right)-\left(g_{i}^{\prime}\right) \in$ $T_{p} G_{2}(K)$, and hence $f^{\omega}\left(\left(g_{i}\right)\right)-f^{\omega}\left(\left(g_{i}^{\prime}\right)\right) \in f^{\omega}\left(T_{p} G_{2}(K)\right)$. Let us prove now that the kernel of this map is $p^{\infty} G_{1}(K)$ : if $g \in p^{\infty} G_{1}(K)$, we can choose $\left(g_{i}\right) \in T_{p}\left(G_{1}(K), g\right)$, which is sent to 0 by $f^{\omega}$. Conversely, assume that, for some $\left(g_{i}\right) \in T_{p}\left(G_{2}(K), g\right)$ and some $\left(h_{i}\right) \in T_{p} G_{2}(K), f^{\omega}\left(\left(g_{i}\right)\right)=f^{\omega}\left(\left(h_{i}\right)\right)$. Then $\left(g_{i}-h_{i}\right) \in T_{p}\left(G_{1}(K), g\right)$, which gives that $g \in p^{\infty} G_{1}(K)$.

Hence we have obtained an embedding $\phi:\left(G_{1}(K) \cap p^{\infty} G_{2}(K)\right) / p^{\infty} G_{1}(K) \hookrightarrow$ $T_{p} G_{3}(K) / f^{\omega}\left(T_{p} G_{2}(K)\right)$. It remains to prove that it is surjective. For $\left(h_{i}\right)_{i \in \mathbb{N}} \in T_{p} G_{3}(K)$, we can realize in $K$ (which is $\omega_{1}$-saturated) the following type of length $\omega$ over $K_{0}\left(\left(h_{i}\right)\right)$ ( $K_{0}$ is a countable subfield of definition):

$$
\bigwedge_{i \in \mathbb{N}}\left(x_{i} \in G_{2} \wedge f\left(x_{i}\right)=h_{i} \wedge x_{i}=[p] x_{i+1}\right)
$$

(It can be realized for $i \leqslant n$ by choosing some $g_{n+1} \in G_{2}(K)$ such that $f\left(g_{n+1}\right)=h_{n+1}$, and then defining $g_{i}=\left[p^{n+1-i}\right] g_{n+1}$.) For a realization $\left(g_{i}\right)_{i \in \mathbb{N}}$ of this type, we have $g_{0} \in G_{1}(K)$ (since $f\left(g_{0}\right)=h_{0}=0$ ) and $\left(g_{i}\right) \in T_{p}\left(G_{2}(K), g_{0}\right)$; hence $g_{0} \in p^{\infty} G_{2}(K)$ and $f^{\omega}\left(\left(g_{i}\right)\right)=\left(h_{i}\right)$.

Remark 4.11. It follows in particular that the sequence $0 \rightarrow G_{1}^{\sharp} \rightarrow G_{2}^{\sharp} \rightarrow G_{3}^{\sharp} \rightarrow 0$ is exact if and only if the map $f^{\omega}: T_{p} G_{2}(K) \rightarrow T_{p} G_{3}(K)$ is surjective.

Proposition 4.12. Let $0 \rightarrow G_{1} \rightarrow G_{2} \rightarrow G_{3} \rightarrow 0$ be an exact sequence of ordinary semiabelian varieties over $K$. Suppose that $G_{1}$ and $G_{3}$ descend to the constants of $K$.

Then, the sequence $0 \rightarrow G_{1}^{\sharp} \rightarrow G_{2}^{\sharp} \rightarrow G_{3}^{\sharp} \rightarrow 0$ remains exact if and only if $G_{2}$ also descends to the constants.

Proof. Here again we may assume that the map $G_{1} \rightarrow G_{2}$ is the inclusion. First, note that the $\sharp$ sequence is exact if and only if $G_{1}(K) \cap G_{2}^{\sharp}=G_{1}^{\sharp}$, by Lemma 3.4.

Let $K_{0}$ be a countable elementary submodel of $K$ over which everything is defined. By isomorphism, we can suppose that both $G_{1}$ and $G_{3}$ are actually defined over $\mathcal{C} \cap K_{0}$, the field of constants of $K_{0}$ (precisely, $G_{i}=\left(G_{i}^{\prime}\right)_{K}$ for some $G_{i}^{\prime}$ over $\mathcal{C} \cap K_{0}, i=1,3$ ). 
If $G_{2}$ descends to the constants, then, by isomorphism, we can suppose that $G_{2}=\left(G_{2}^{\prime}\right)_{K}$ for some $G_{2}^{\prime}$ over the constants, so, for every $i, G_{i}{ }^{\sharp}=G_{i}^{\prime}(\mathcal{C})$. And then $G_{1}(K) \cap G_{2}^{\sharp}=$ $G_{1}^{\prime}(K) \cap G_{2}^{\prime}(\mathcal{C})=G_{1}^{\prime}(\mathcal{C})=G_{1}^{\sharp}$.

For the converse, suppose that $0 \rightarrow G_{1}^{\sharp} \rightarrow G_{2}^{\sharp} \rightarrow G_{3}^{\sharp} \rightarrow 0$ is exact.

Our assumption that the $G_{i}$ are ordinary ensures that, for each $i, T_{p} G_{i}(\bar{K}) \cong \mathbb{Z}_{p}{ }^{a_{i}}$, where $a_{i}$ is the dimension of the abelian part of $G_{i}$. If $G_{1}$ and $G_{3}$ descend to $\mathcal{C}$, then $T_{p} G_{1}(K)=T_{p} G_{1}(\mathcal{C})=T_{p} G_{1}(\bar{K})$ and $T_{p} G_{3}(K)=T_{p} G_{3}(\mathcal{C})=T_{p} G_{3}(\bar{K})$. By Remark 4.11, the sequence

$$
0 \longrightarrow T_{p} G_{1}(K) \longrightarrow T_{p} G_{2}(K) \longrightarrow T_{p} G_{3}(K) \longrightarrow 0
$$

is exact. It follows that $T_{p} G_{2}(K) \cong \mathbb{Z}_{p}{ }^{a_{1}+a_{3}}$. As $a_{1}+a_{3}=a_{2}$ (by exactness of $0 \longrightarrow$ $\left.G_{1} \longrightarrow G_{2} \longrightarrow G_{3} \longrightarrow 0\right)$, and as $T_{p} G_{2}(K)$ is a direct factor submodule of $T_{p} G_{2}(\bar{K})$, it follows that $T_{p} G_{2}(K)=T_{p} G_{2}(\bar{K})$, and, by Proposition 4.3 , that $G_{2}$ descends to the constants.

Corollary 4.13. For any ordinary abelian variety $A$ defined over the constants of $K$, there exists an exact sequence over $K$,

$$
0 \longrightarrow \mathbb{G}_{m} \longrightarrow H \longrightarrow A_{K} \longrightarrow 0,
$$

such that

$$
0 \longrightarrow \mathbb{G}_{m}^{\sharp} \longrightarrow H^{\sharp} \longrightarrow\left(A_{K}\right)^{\sharp} \longrightarrow 0
$$

is not exact.

Proof. We use the fact that $\operatorname{EXT}\left(A, \mathbb{G}_{m}\right)$ is parameterized (up to isomorphism) by the dual abelian variety of $A$, say $\hat{A}$, which is also over the constants, as in Proposition 2.7. Then $H$ will descend to the constants $\mathcal{C}$ of $K$ if and only if $H$ corresponds to a $\mathcal{C}$-rational point of $\hat{A}$. So just pick some $K$-rational point of $\hat{A}$ which is not $\mathcal{C}$-rational.

We have established in Proposition 4.9 the connection between exactness and relative Morley rank, and we can conclude that the following holds.

Corollary 4.14. There is an ordinary semiabelian variety $G$, such that $G^{\sharp}$ does not have relative Morley rank.

In fact, as above, for any ordinary abelian variety $A$ defined over $K^{p^{\infty}}$, there is some semiabelian variety $G$ in $\operatorname{EXT}\left(A, \mathbb{G}_{m}\right)$ such that $G^{\sharp}$ does not have relative Morley rank.

We will finish this section with some direct corollaries of Proposition 4.10. Again, $0 \longrightarrow G_{1} \longrightarrow G_{2} \stackrel{f}{\longrightarrow} G_{3} \longrightarrow 0$ is an exact sequence of semiabelian varieties over $K$, with $G_{1} \rightarrow G_{2}$ the inclusion map. Recall from Proposition 4.10 that $\left(G_{1}(K) \cap G_{2}^{\sharp}\right) / G_{1}^{\sharp} \cong$ $T_{p} G_{3}(K) / f^{\omega}\left(T_{p} G_{2}(K)\right)$.

Corollary 4.15. If $G_{3}\left[p^{\infty}\right](K)$ is finite, then the $\sharp$ sequence is exact.

Proof. Since $G_{3}\left[p^{\infty}\right](K)$ is finite, $T_{p} G_{3}(K)=0$.

If we add the assumption that the semiabelian varieties have relative Morley rank, we get the following characterization. 
Proposition 4.16. Let $0 \longrightarrow G_{1} \longrightarrow G_{2} \longrightarrow G_{3} \longrightarrow 0$ be an exact sequence of semiabelian varieties over $K$ such that $G_{2}^{\sharp}$ has relative Morley rank. Then the following are equivalent.

(1) The sequence $0 \longrightarrow G_{1}{ }^{\sharp} \longrightarrow G_{2}{ }^{\sharp} \longrightarrow G_{3}{ }^{\sharp} \longrightarrow 0$ is exact.

(2) $G_{1}\left[p^{\infty}\right](K) \cap G_{2}^{\sharp}=G_{1}\left[p^{\infty}\right](K) \cap G_{1}^{\sharp}$.

In particular, the $\sharp$ sequence will be exact when $G_{1}$ descends to the constants, or, more generally, when $G_{1}\left[p^{\infty}\right](\bar{K})=G_{1}\left[p^{\infty}\right](K)$, and also when $G_{1}\left[p^{\infty}\right](K)=0$.

Proof. Recall that $G_{i}^{\sharp}=p^{\infty} G_{i}(K)$. We know that (1) holds if and only if $G_{1}^{\sharp}=G_{1}(K) \cap$ $G_{2}{ }^{\sharp}$. So, trivially, (1) implies (2). We know that $G_{1}^{\sharp}$ contains all the $p^{\prime}$-torsion of $G_{1}(K)$. It follows that, if $(2)$ holds, then $\left(G_{1}(K) \cap G_{2}^{\sharp}\right) / G_{1}^{\sharp}$ is torsion free. As by assumption $G_{2}{ }^{\#}$ has relative Morley rank, this quotient must be finite; if it is torsion free, it is trivial.

If $G_{1}\left[p^{\infty}\right](\bar{K})=G_{1}\left[p^{\infty}\right](K)$, then $G_{1}\left[p^{\infty}\right](K) \subset G_{1}^{\sharp}$, and the conclusion holds.

\subsection{Further examples}

We will see in $\S 5.3$ that, in characteristic 0 , the $\sharp$ functor preserves exact sequences of abelian varieties. This is not the case in characteristic $p$, even for ordinary abelian varieties.

The examples of nonexactness for abelian varieties will have to be quite different from the examples seen in the previous section for semiabelian varieties, as can be seen from the following direct corollary of Proposition 4.16. Recall from Fact 3.8 that, for all abelian varieties $A, A^{\sharp}$ has finite relative Morley rank.

Corollary 4.17. We assume that $K$ is $\omega_{1}$-saturated. Let $0 \longrightarrow C \longrightarrow B \longrightarrow A \longrightarrow 0$ be an exact sequence of abelian varieties over $K$. If $C(K)$ has no p-torsion, or if $C$ descends to the constants, then the sequence $0 \longrightarrow C^{\sharp} \longrightarrow B^{\sharp} \longrightarrow A^{\sharp} \longrightarrow 0$ is exact.

Remark 4.18. From Corollary 4.17 and the example given in Remark 4.20, we see that Proposition 4.12 does not hold for nonordinary (semi)abelian varieties.

There are still cases, not covered by Corollary 4.17, where one obtains nonexactness, even in the ordinary case.

Proposition 4.19. There is an exact sequence of (ordinary) abelian varieties such that the induced $\sharp$ sequence is not exact.

Proof. Let $A$ be an ordinary elliptic curve, defined over $K^{p}$, which does not descend to $K^{p^{\infty}}$, and let $C$ be an ordinary elliptic curve defined over $K^{p^{\infty}}$. Then we know by Proposition 4.3 that $A[p](K) \cong \mathbb{Z} / p \mathbb{Z} \cong C[p](K)$ but $A\left[p^{\infty}\right](K)$ is finite. Pick an isomorphism $f$ between $A[p](K)$ and $C[p](K)$.

Let $H \subset A[p](K) \times C[p](K):=\{(a,-f(a)): a \in A \mid p][K)\}$, and $B:=\left(A_{K} \times C_{K}\right) / H$. Then $A_{K}$ is isomorphic to $A_{1}:=\left(A_{K} \times 0+H\right) / H \subset B$. Consider the exact sequence

$$
0 \longrightarrow A_{1} \longrightarrow B \stackrel{g}{\longrightarrow} B / A_{1} \longrightarrow 0 .
$$

Note that $C_{1}:=B / A_{1}$ is isogenous to $C_{K}$; hence, by 2.17 or 4.5 , it descends to $K^{p^{\infty}}$. 
One can check that the $p^{\infty}$ sequence is no longer exact: more precisely, that $p^{\infty} A_{1}(K) \neq$ $A_{1}(K) \cap p^{\infty} B(K)$.

Furthermore, if $K$ is $\omega_{1}$-saturated, by applying Proposition 4.10, one sees that $\left(A_{1}(K) \cap\right.$ $\left.p^{\infty} B(K)\right) / p^{\infty} A_{1}(K)$ is isomorphic to $\mathbb{Z} / p \mathbb{Z}$.

Remark 4.20. The following example illustrates the necessity of the separable hypothesis in Facts 2.15 and 2.16, and of the ordinary hypothesis in Corollary 4.6 and Proposition 4.12.

Let $E$ be a supersingular elliptic curve over $K$ ( $\omega_{1}$-saturated), necessarily descending to $\overline{\mathbb{F}_{p}}$. For any abelian variety $A$ there is a one-to-one correspondence between (isomorphism classes of) purely inseparable isogenies and sub $p$-Lie algebras of Lie $A$ (see [33] or [26]). It follows that there is an abelian variety $A$ over $K$, isogenous to $E \times E$, which cannot be isomorphic to any abelian variety defined over $\overline{\mathbb{F}_{p}}$.

Furthermore, for such an $A$, it is easily seen that $A$ lies in $\operatorname{EXT}\left(E_{1}, E_{2}\right)$ for some elliptic curves $E_{1}$ and $E_{2}$ descending to $\overline{\mathbb{F}_{p}}$, and in this case $0 \rightarrow E_{1}^{\sharp} \rightarrow A^{\sharp} \rightarrow E_{2}^{\sharp} \rightarrow 0$ is exact by Corollary 4.17 .

We thank A. Chambert-Loir and L. Moret-Bailly for pointing out these arguments to us.

We finish this section with a summary in the case of semiabelian varieties over $K$ $\left(\omega_{1}\right.$-saturated $)$

$$
0 \longrightarrow T \longrightarrow G \longrightarrow E \longrightarrow 0
$$

with $E$ an elliptic curve.

Proposition 4.21. Let $G$ be as above.

(i) If $E$ is supersingular, then the $\sharp$ sequence remains exact, and $G^{\sharp}$ has relative Morley rank.

(ii) If $E$ is ordinary and does not descend to the constants, then the $\sharp$ sequence remains exact, and $G^{\sharp}$ has relative Morley rank.

(iii) If $E$ is ordinary and descends to the constants, the following are equivalent.

- The $\sharp$ sequence is exact.

- $G$ descends to the constants.

- $G^{\sharp}$ has relative Morley rank.

- $G\left[p^{\infty}\right](K)$ is infinite.

In the case when $G$ does not descend to the constants, then $\left(T(K) \cap G^{\sharp}\right) / T^{\sharp}$ is isomorphic to the profinite group $\mathbb{Z}_{p}$.

Proof. Recall first that Proposition 4.9 says that in the present context $G^{\sharp}$ has relative Morley rank if and only if the $\sharp$ sequence is exact.

(i) If $E$ is supersingular, it has no $p$-torsion, and Corollary 4.15 applies.

(ii) If $E$ does not descend to the constants, Corollary 4.15 applies. 
(iii) If $E$ is ordinary and descends to $K^{p^{\infty}}$, by Proposition 4.12 , the $\sharp$ sequence will be exact if and only if $G$ descends to $K^{p^{\infty}}$. As $T$ has no $p$-torsion, $G\left[p^{\infty}\right](\bar{K}) \cong$ $E\left[p^{\infty}\right](\bar{K}) \cong \mathbb{Z}_{p^{\infty}}$. So, if $G$ descends to the constants, then $G\left[p^{\infty}\right](K)=G\left[p^{\infty}\right](\bar{K})$, and so is infinite.

If $G$ does not descend to $K^{p^{\infty}}$, using Proposition 4.3 , there is some $n \geqslant 1$ such that $G\left[p^{\infty}\right](K)=G\left[p^{n}\right](K)$, and hence is finite.

In particular, in this case, $T_{p} G(K)=\{0\}$. By Proposition 4.10, $\left(T(K) \cap G^{\sharp}\right) / T^{\sharp}$ is isomorphic to $T_{p} E(K) / \tilde{f}^{\omega}\left(T_{p} G(K)\right) \cong T_{p} E(K) \cong \mathbb{Z}_{p}$, completing the proof of (iii).

\section{Uniform results in all characteristics}

In order to prove the analogues of Proposition 4.12 and Corollary 4.13 in characteristic 0, we need to use more differential algebraic methods, and in particular D-structures. But in fact the elementary proofs we gave in the previous section for the characteristic $p$ case can also be seen as involving D-structures and being similar to the characteristic 0 case. This was just 'hidden' by the fact that the objects manipulated have, in characteristic $p$, an easy algebraic description. We believe it is interesting though to explain this uniformity precisely, and in order to do this we will need to introduce D-structures on group schemes.

But before we launch into this slightly dry exposition, let us point out that most of the 'uniform' results can in fact be read at the 'analogy' level, without actually understanding the D-structure in the characteristic $p$ case. This will be briefly explained at the beginning of $\S 5.2$.

For the whole of this section, $(K, \partial)$ will be a model of $\mathrm{DCF}_{0}$ or $\mathrm{CHF}_{p, 1}$, where in the latter case we assume $\omega_{1}$-saturation.

\subsection{D-structures and descent}

A good exposition of notions presented here can be found in [18]; one can also look at [3].

Definition 5.1. 1. An (iterative) Hasse D-structure on a scheme $X$ over $K$ is an (iterative) Hasse derivation $\partial$ on the structure sheaf $\mathcal{O}_{X}$, which means that, for each open subset $U \subset X$, we have an (iterative) Hasse derivation $\partial_{U}: \mathcal{O}_{X}(U) \rightarrow$ $\mathcal{O}_{X}(U)$, such that the structure homomorphism $K \rightarrow \mathcal{O}_{X}(U)$ and the restriction homomorphisms $\mathcal{O}_{X}\left(U^{\prime}\right) \rightarrow \mathcal{O}_{X}(U)$ preserve the Hasse derivations.

2. A morphism of schemes with (iterative) D-structure $\left(X, \partial_{X}\right) \rightarrow\left(Y, \partial_{Y}\right)$ is a morphism of schemes $X \rightarrow Y$ such that the corresponding morphism of sheaves preserves the Hasse derivations.

3. In particular, for $(R, \partial)$ an (iterative) Hasse differential algebra over $(K, \partial)$, $(\operatorname{Spec}(R), \partial)$ is a scheme with an (iterative) D-structure, and a D-point of $\left(X, \partial_{X}\right)$ with value in $R$ is by definition a morphism of schemes with (iterative) D-structure $(\operatorname{Spec}(R), \partial) \rightarrow\left(X, \partial_{X}\right)$. We denote this set of D-points by $\left(X, \partial_{X}\right)^{\partial}(R)$.

4. If $\left(X, \partial_{X}\right)$ is a scheme with an (iterative) D-structure and $Y$ is a closed subscheme of $X$, we say that $Y$ is an (iterative) D-subscheme of $\left(X, \partial_{X}\right)$ if $\partial_{X}$ induces an (iterative) Hasse derivation on $\mathcal{O}_{Y}$, or, equivalently, if the sheaf of ideals $\mathcal{I}_{Y} \subset \mathcal{O}_{X}$ is a sheaf 
of D-ideals (i.e., for each open subset $U \subset X, \mathcal{I}_{Y}(U)$ is an ideal of $\mathcal{O}_{X}(U)$ stable by $\left.\partial_{U}\right)$.

5. We say that $(G, \partial)$ is a group scheme with an (iterative) Hasse derivation if $G$ is a group scheme over $K$, with an (iterative) D-structure $\partial$, such that the identity element is a D-point with value in $K$, and such that the inverse map and the multiplication map are morphisms of schemes over $K$ with (iterative) D-structure.

Remark 5.2. For this last point, we have used the fact that, if $\left(X, \partial_{X}\right)$ and $\left(Y, \partial_{Y}\right)$ are schemes with an (iterative) Hasse derivation over $K, X \times_{K} Y$ can be endowed in a unique way with an (iterative) Hasse derivation such that the projection maps are morphisms in this category. This is a straightforward consequence of the existence of tensor products in the category of (iterative) Hasse differential algebras over $K$.

In the case of algebraic groups over $K$, we can give another description of (iterative) D-structures, which uses the notion of prolongations. The two approaches coincide; see [1] or [18].

We first recall the description of the prolongations for Hasse derivations, given in the greatest generality in [25] (see also [11] or [37]).

If $V$ is a smooth irreducible algebraic variety over $K$, the $n$th prolongation of $V$ is an algebraic variety $\Delta_{n} V$ over $K$ characterized as follows. For any $K$-algebra $\phi: K \rightarrow R$, the set of $R$-points of $\Delta_{n} V$ is $\Delta_{n} V(R)=V\left(R^{(n)}\right)$, where $R^{(n)}=R[X] /\left(X^{n+1}\right)$ is endowed with the structure of a $K$-algebra by the Taylor map $K \rightarrow R^{(n)}, a \mapsto \sum_{i=0}^{n} \phi\left(\partial_{i}(a)\right) X^{i}$.

For example, if $V \subseteq \mathbb{A}^{n}$ is a smooth irreducible affine variety, then $\Delta_{n} V$ can be described as the Zariski closure of the image of $V(K)$ by $\partial_{\leqslant n}:=\left(\partial_{0}, \ldots, \partial_{n}\right)$,

$$
\Delta_{n} V:=\overline{\left\{\partial_{\leqslant n}(x): x \in V(K)\right\}} \subseteq \mathbb{A}^{m n+1}
$$

In general, using the Taylor map $K \rightarrow K^{(n)}$, we get a (definable) map $\partial_{\leqslant n}: V(K) \longrightarrow$ $\Delta_{n} V(K)$, having Zariski-dense image. For $m \geqslant n \geqslant 0$, we have a natural projection morphism $\pi_{m, n}: \Delta_{m} V \longrightarrow \Delta_{n} V$ such that $\pi_{m, n} \circ \partial_{\leqslant m}=\partial_{\leqslant n}$.

These constructions are functorial, and, in the case where $V=G$ is a connected algebraic group, each $\Delta_{n} G$ has a natural structure of an algebraic group, and the maps $\partial_{\leqslant n}, \pi_{m, n}$ are homomorphisms.

Fact 5.3. Let $G$ be a connected algebraic group over $K$. There is a bijective correspondence between the D-structures on the group scheme $G$ and the sequences of homomorphic regular sections $s=\left(s_{n}\right)_{n \in \mathbb{N}}$ for the projective system $\left(\pi_{m, n}: \Delta_{m} G \longrightarrow \Delta_{n} G\right)_{m \geqslant n \geqslant 0}$ (i.e., we require that each $s_{n}: G \longrightarrow \Delta_{n} G$ is a regular homomorphism over $K$, and that these homomorphisms satisfy $\pi_{m, n} \circ s_{m}=s_{n}$ and $\left.s_{0}=i d_{G}\right)$.

The condition for a D-structure to be iterative translates into obvious, but laborious to write out, conditions on the corresponding sequence of sections (see [1] or [18]).

For $(G, \partial)$ a connected algebraic group with a D-structure s over $K$, the corresponding sequence of sections, and $(L, \partial)$ an extension of $K$, the set of D-points can be described 
as the $\mathrm{M}$-definable subgroup of $G(L)$ :

$$
(G, \partial)^{\partial}(L)=\left\{x \in G(L): \partial_{\leqslant n}(x)=s_{n}(x) \text { for all } n \geqslant 0\right\} .
$$

Moreover, if $L$ is a model ( $\omega_{1}$-saturated in characteristic $\left.p\right)$, then $(G, \partial)^{\partial}(L)$ is Zariski dense in $G$, and it has transcendence degree equal to the dimension of $G$.

Remark 5.4. Let $G$ be a semiabelian variety over $K$. In order to define an iterative D-structure on $G$, it suffices that, for some (any) generic point $g$ of $G^{\sharp}(L)$ over $K$ ( $L$ an elementary extension of $K$ ), for any $n \geqslant 0, \partial_{n}(g) \in K(g)$. Indeed, because $G^{\sharp}$ is Zariski dense in $G$, the existence of such a point $g$ induces a rational map from $G(L)$ to $\Delta_{n} G(L)$, which can be extended to a homomorphism $s_{n}$ by a classical stability argument. We obtain in this way a D-structure on $G$ because $s_{n}$ coincides with $\partial_{\leqslant}$on the Zariski-dense subgroup $G^{\sharp}$, and the $\partial_{\leqslant n}$ give a sequence of definable sections by definition. The iterativity comes from the iterativity of $\partial$, because, on an affine open subset $U$, the Hasse derivation given by $\left(s_{n}\right)$ is such that $\left(\operatorname{Frac}\left(\mathcal{O}_{G}(U)\right), D\right)$ is isomorphic to $(K(g), \partial)$, which is an iterative Hasse field.

In particular, if $G$ is defined over the constants $\mathcal{C}$, for each $g \in G^{\sharp}=G(\mathcal{C}), \partial_{n}(g)=0$ for $n \geqslant 1$; hence we can define a natural iterative D-structure on $G$. The two following results are a converse of this observation.

Fact 5.5. Let $G$ be a connected algebraic group over $K$. Then, for each $n \geqslant 0$, the kernel of $\pi_{n, 0}: \Delta_{n} G \longrightarrow G$ is a unipotent group (see [28] in characteristic 0 or [1] in arbitrary characteristic). It follows that a semiabelian variety $G$ over $K$ admits at most one D-structure, since the difference between two sections is a homomorphism $G \longrightarrow \operatorname{Ker}\left(\pi_{n, 0}\right)$, and hence zero.

Proposition 5.6. Let $G$ be a semiabelian variety over $K$ with an iterative $D$-structure. Then $G$ descends to the constants.

Proof. In the characteristic 0 case, this result appears implicitly in [10]; see Lemma 3.4 in [6] for more explanations.

In the characteristic $p$ case, it is proved in [4] (Proof of Theorem 4.4) that such a semiabelian variety $G$ descends to $K^{p^{n}}$ for every $n$. Then Proposition 2.7 applies.

Note that, in characteristic 0 , since an iterative Hasse derivation satisfies $\partial_{i}=\frac{1}{i !} \partial_{1}$, it suffices to have a usual derivation $D_{1}$ on $\mathcal{O}_{G}$, or equivalently a section $s=s_{1}: G \longrightarrow \Delta_{1} G$ in order to define an iterative D-structure; $\Delta_{1} G$ is also known as the twisted tangent bundle of $G$.

We will now state the criteria for descent which we will be using.

In the characteristic 0 case we quote from [6], 33.1 , and, in characteristic $p$, this is the object of $\S 5.2$.

(Characteristic 0 ) Let $G$ be a semiabelian variety over $K$, and let $\tilde{G}$ denote the universal extension of $G$ by a vector group (as defined in [31]). Let us write $\tilde{G}$ as

$$
0 \longrightarrow W_{G} \longrightarrow \tilde{G} \longrightarrow G \longrightarrow 0,
$$

where $W_{G}$ is a vector group. 
Fact 5.7. $\quad$ 1. $\tilde{G}$ admits a unique iterative D-structure.

2. Consider $U_{G}$, the maximal subgroup of $W_{G}$, which is a D-subgroup of $(\tilde{G}, \partial)$. We still denote by $\partial$ the $D$-structure induced on $\tilde{G} / U_{G}$. Then $G^{\sharp}$ is isomorphic to $\left(\tilde{G} / U_{G}, \partial\right)^{\partial}(K)$.

3. It follows from Proposition 5.6 that $G$ descends to the constants if and only if $G \simeq$ $\tilde{G} / W_{G}$ has a D-structure if and only if $U_{G}=W_{G}$ (since in this case the projection $\tilde{G} \rightarrow \tilde{G} / W_{G}$ must preserve the D-structures; see Corollary 3.6 in [6]).

4. Furthermore, the functor of D-points is exact on the class of algebraic D-groups [17]. In particular, $\left(\tilde{G} / U_{G}, \partial\right)^{\partial}(K) \cong(\tilde{G}, \partial)^{\partial}(K) /\left(U_{G}, \partial\right)^{\partial}(K)$.

\subsection{D-structure on $\tilde{G}$ in characteristic $p$}

In this $\operatorname{section}, \operatorname{char}(K)=p$.

In characteristic $p$, the universal extension of $G$ by a vector group does not in general have an iterative $D$-structure. Indeed, if $G$ is an arbitrary semiabelian variety, $(H, D)$ any connected algebraic $K$-group with an iterative D-structure, and $f$ a separable morphism from $H$ onto $G$, then $G$ must be isogenous to $\left(G_{0}\right)_{K}$ for some $G_{0}$ semiabelian variety over the constants: $f$ maps $(H, D)^{\partial}(L)$ onto $G^{\sharp}(L)$ by density ( $L$ a sufficiently saturated extension of $K$ ), and it follows that $K(\{g\})$ is finitely generated over $K$ as a field where $g$ is a generic point of $G^{\sharp}(L)$, which implies the conclusion by an argument given in [4] (compare with Remark 5.4 and Proposition 5.6). This explains why the introduction of group schemes (or proalgebraic groups) with D-structures will be unavoidable in a uniform treatment of both characteristics.

The construction we describe below is, as we mentioned before, the D-structure argument which lies behind the simple algebraic treatment we gave in $\S 4.2$. But, as also mentioned at the beginning of $\S 5$, most of the 'uniform' results can in fact be read at the 'analogy' level, without actually understanding the D-structure in the characteristic $p$ case.

More precisely:

Recall from $\S 4$ that, if $G$ is a semiabelian variety over $K$,

$$
\tilde{G}:=\lim _{\leftarrow}\left(G_{0} \stackrel{[p]}{\longleftarrow} G_{1} \stackrel{[p]}{\longleftarrow} \cdots\right) .
$$

Denote $T_{p} G$ by $W_{G}, T_{p} G(K)$ by $\left(U_{G}, \partial\right)^{\partial}(K)$, and $\tilde{G}(K)$ by $(\tilde{G}, \partial)^{\partial}(K)$. These are $*$-definable groups in $K$. From $\S 4$, we know that

$$
G^{\sharp} \text { is isomorphic to }(\tilde{G}, \partial)^{\partial}(K) /\left(U_{G}, \partial\right)^{\partial}(K) \text {. }
$$

Then one can more or less jump to $\S 5.3$ and read the statements and proofs of Lemma 5.19 and Proposition 5.20, as they are, with the above definitions for the characteristic $p$ case. Except for condition (iv) in Proposition 5.20, which then makes sense only in characteristic 0 .

We will now begin the real construction.

We have $G$ and $\tilde{G}$ as above. The (scheme-theoretic) kernel of the projection $\pi: \tilde{G} \rightarrow G_{0}$ is the Tate module $T_{p} G$. From now on, it is important to consider $\tilde{G}$ and $T_{p} G$ as group 
schemes. We will denote by $X_{0}$ a system of coordinates for $G_{0}$ (for a fixed affine covering say), such that $X_{0}$ generates the maximal ideal of the identity element of $G_{0}$, and by $X_{i}$ its image in $\mathcal{O}_{G_{i}}$ by the identity isomorphism. It follows that the sections of the sheaf

$$
\mathcal{O}_{\tilde{G}}=\lim _{\rightarrow}\left(\mathcal{O}_{G_{0}} \stackrel{[p]^{*}}{\rightarrow} \mathcal{O}_{G_{1}} \cdots\right)
$$

are generated over $K$ by $\left(X_{i}\right)_{i \in \mathbb{N}}$.

Here are some definitions which will play a role in the construction of the D-structure on $\tilde{G}$ in the proof of Proposition 5.8. The map $[p]^{*}$ induced by $[p]$ on $\mathcal{O}_{\tilde{G}}$ is given by $[p]^{*}\left(X_{0}\right)=[p]_{G_{0}}^{*}\left(X_{0}\right)$ and $[p]^{*}\left(X_{i}\right)=X_{i-1}$ for $i \geqslant 1$. We can define a 'shift' homomorphism $s$ on $\tilde{G}$ characterized by $s^{*}\left(X_{i}\right)=X_{i+1}$. It is clear that $s \circ[p]=i d_{\tilde{G}}$, and that $s$ and $[p]$ commute.

At the level of points, for $\left(g_{0}, g_{1}, g_{2}, \ldots\right) \in \tilde{G}(L), L$ an extension of $K, s\left(g_{0}, g_{1}, g_{2}, \ldots\right)$ $=\left(g_{1}, g_{2}, g_{3}, \ldots\right)$ and $[p]\left(g_{0}, g_{1}, g_{2}, \ldots\right)=\left([p] g_{0}, g_{0}, g_{1}, \ldots\right)$.

Now, for each $n$, we have $\left[p^{n}\right]_{\tilde{G}}=V_{n} \circ F^{n}$, where $F^{n}: \tilde{G} \rightarrow \tilde{G}^{\left(p^{n}\right)}$ is the power of the Frobenius homomorphism and $V_{n}: \tilde{G}^{\left(p^{n}\right)} \rightarrow \tilde{G}$ the $n$th Verschiebung (induced by the Verschiebung on each $G_{i}$ ).

Proposition 5.8. There exists an iterative D-structure on $\tilde{G}$. Moreover, this D-structure is unique 'in a strong sense'.

Proof. We first state the uniqueness in a strong sense: for any homomorphism of K-algebras $D_{0}: \mathcal{O}_{\tilde{G}} \rightarrow A$ (strictly speaking, we should replace $\mathcal{O}_{\tilde{G}}$ by its ring of sections for some open set), there is at most one (noniterative) Hasse derivation from $\mathcal{O}_{\tilde{G}}$ to $A$ over $K$ extending $D_{0}$. By this we only mean a sequence of additive maps $\left(D_{i}: \mathcal{O}_{\tilde{G}} \rightarrow A\right)_{i \in \mathbb{N}}$ satisfying the generalized Leibniz rule and agreeing with $\partial$ on $K$ (we cannot require iterativity at this level of generality since $\left.A \neq \mathcal{O}_{\tilde{G}}\right)$.

We assume that we have such a Hasse derivation $\left(D_{i}\right)_{i \in \mathbb{N}}$, and we consider some $f \in \mathcal{O}_{\tilde{G}}$ and some index $i<p^{n}$. Because of the previous equalities, we must have $D_{i}(f)=D_{i}\left(F^{n *} \circ V_{n}^{*} \circ s^{n *}(f)\right)$. But $F^{n *}\left(V_{n}^{*} \circ s^{n *}(f)\right)$ can be represented locally as a rational function of the variables $X_{j}^{p^{n}}$, and hence there is a unique possible value for $D_{i}(f)$ because, if $P$ is a polynomial with coefficients in $K, D_{i}\left(P\left(X^{p^{n}}\right)\right)=P^{\partial_{i}}\left(D_{0}(X)^{p^{n}}\right)$, where $P^{\partial_{i}}$ is obtained by applying $\partial_{i}$ to the coefficients of $P$.

Now, we start to define a truncated Hasse derivation on $\mathcal{O}_{\tilde{G}}$. Since $\tilde{G}^{\left(p^{n}\right)}$ descends to $K^{p^{n}}$, on which $\partial_{<p^{n}}$ is trivial, we obtain a truncated Hasse derivation $D_{<p^{n}}^{\prime}$ on $\mathcal{O}_{\tilde{G}^{\left(p^{n}\right)}}=$ $\mathcal{O}_{G^{\prime}} \otimes_{K p^{n}} K\left(G^{\prime}\right.$ is a model of $\tilde{G}^{\left(p^{n}\right)}$ over $\left.K^{p^{n}}\right)$ by putting the trivial truncated Hasse derivation on $\mathcal{O}_{G^{\prime}}$. Now, we define

$$
D_{<p^{n}}=F^{n *} \circ D_{<p^{n}}^{\prime} \circ V_{n}^{*} \circ s^{n *} .
$$

It is clear that $D_{<p^{n}}^{\prime}$ preserves the comultiplication and coinverse of $G^{\prime}$ because these are $K^{p^{n}}$-morphisms; hence $D_{<p^{n}}$ preserves the group structure of $\tilde{G}$. And, because of the uniqueness that we have noticed before, the $D_{<p^{n}}$ for different values of $n$ are compatible, and hence we have defined a (the unique) D-structure $D$ on $\tilde{G}$. It is actually iterative 
since the $D_{<p^{n}}^{\prime}$ are (as tensor products of the trivial iterative derivation and $\partial$ ) and since $V_{n}^{*} \circ s^{n *} \circ F^{n *}=i d$ (because $\left.F^{n} \circ s^{n} \circ V_{n}=F^{n}\left(s^{n}\right) \circ F^{n} \circ V_{n}=F^{n}\left(s^{n} \circ\left[p^{n}\right]_{\tilde{G}}\right)=i d\right)$.

Remark 5.9. Let us give a slightly informal description of this D-structure in terms of sections $s_{n}: \tilde{G} \rightarrow \Delta_{n} \tilde{G}$. For instance, $s_{1}: \tilde{G} \rightarrow \Delta_{1} \tilde{G}=\stackrel{\lim }{\leftarrow} \Delta_{1} G_{i}$ is given by the sequence of homomorphisms $\left(s_{1 i}\right)_{i}$ :

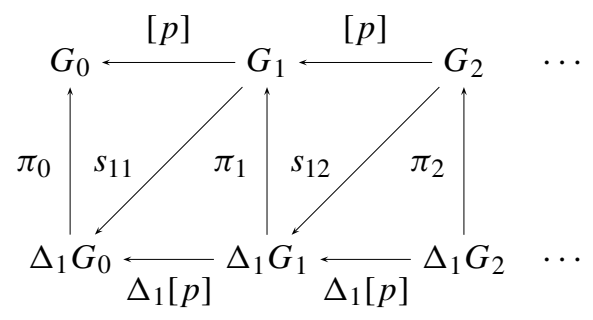

where, if $x_{i} \in G_{i}(K)(i \leqslant 1)$,

$$
s_{1 i}\left(x_{i}\right)=\left([p] x_{i}, V^{\partial_{1}} \circ F\left(x_{i}\right)\right) \in \Delta_{1} G_{i-1}(K) .
$$

As in the proof of Proposition 5.8, $V^{\partial_{1}}$ is defined so that, for $K$-rational points, $V^{\partial_{1}}(F(x))=\partial_{1}(V(F(x)))$ (recall that $F(x)$ is a constant for $\partial_{1}$ ), which corresponds to applying $\partial_{1}$ to the coefficients of $V$ when $V$ is a polynomial, with the obvious generalization for rational functions. Let us remark that, for every $\left(a_{i}\right)_{i \in \mathbb{N}} \in \tilde{G}(K)$, $\left(\left(a_{i}\right)_{i},\left(\partial_{1}\left(a_{i}\right)\right)_{i}\right)=s_{1}\left(\left(a_{i}\right)_{i}\right)$; it is actually a general fact, proved in Lemma 5.11.

Remark 5.10. It follows from the uniqueness in the strong sense that, if $\left(X, \partial_{X}\right)$ is a scheme with a D-structure, and $f: X \rightarrow \tilde{G}$ a morphism of schemes, it is automatically a morphism of schemes with a D-structure: for any open subset $U \subseteq \tilde{G}$, the corresponding homomorphism $f_{U}^{*}: \mathcal{O}_{\tilde{G}}(U) \rightarrow \mathcal{O}_{X}\left(f^{-1}(U)\right)$ is such that $\partial_{X} \circ f_{U}^{*}$ and $f_{U}^{*} \circ \partial_{\tilde{G}}$ are two Hasse derivations extending $f_{U}^{*}$, and hence must coincide, which means that $f_{U}^{*}$ is a D-homomorphism.

We now focus on the D-points of $(\tilde{G}, D)$.

Lemma 5.11. Let $(R, \partial)$ be an iterative Hasse differential $K$-algebra. Then $(\tilde{G}, D)^{\partial}(R)=$ $\tilde{G}(R)$. Of course this is still true for every D-subscheme of $(\tilde{G}, D)$.

Proof. The proof is simply Remark 5.10 for the particular case $\left(X, \partial_{X}\right)=(\operatorname{Spec} R, \partial)$.

In Fact 5.7, we defined, in characteristic $0, U_{G}$ to be the maximal subgroup of $W_{G}$ which is a D-subgroup of $(\tilde{G}, D)$. Here is the characteristic $p$ version.

Definition 5.12. We define $U_{G}$ as the maximal closed subscheme of $W_{G}:=T_{p} G$ which is a D-subscheme of $(\tilde{G}, D)$. If we have chosen $X_{0}$ such that it generates the maximal ideal of the identity element of $G_{0}, U_{G}$ is the D-subscheme of $(\tilde{G}, D)$ defined by the sheaf of D-ideals $\mathcal{I}_{U}$ of $\left(\mathcal{O}_{\tilde{G}}, D\right)$ generated by $X_{0}$. We see that $U_{G}$ is actually a group D-subscheme 
of $(\tilde{G}, D)$ because $D$ preserves the group structure, which implies that $\iota\left(\mathcal{I}_{U}\right) \subseteq \mathcal{I}_{U}$ and $\mu\left(\mathcal{I}_{U}\right) \subseteq \mathcal{I}_{U} \otimes \mathcal{I}_{U}$

As in characteristic 0 , we obtain the following.

Lemma 5.13. $G$ descends to the constants if and only if $T_{p} G\left(=W_{G}\right)=U_{G}$.

Proof. The argument is standard. We know that $G$ descends to the constants if and only if it admits an iterative D-structure (see $\S 5.1$ ). If $T_{p} G=U_{G}, T_{p} G$ is a D-subgroup of $\tilde{G}$; hence there is an iterative D-structure on the quotient $\tilde{G} / T_{p} G \simeq G$ (this is done in the characteristic 0 case in [17]; details in characteristic $p$ are worked out in [3]). For the converse, if $G$ descends to the constants, then, up to isomorphism, each $G_{i}$ is endowed with the trivial iterative D-structure, and each $[p]$ map is a D-morphism. It follows that the unique (iterative) D-structure on $\tilde{G}$ is the trivial one, for which $T_{p} G$ is a D-subgroup of $\tilde{G}$, and hence is equal to $U_{G}$.

Lemma 5.14. $U_{G}(K)=\left(U_{G}, D\right)^{\partial}(K)=T_{p} G(K)$.

Proof. By Lemma 5.11, each point in $T_{p} G(K)$ is a D-point of $(\tilde{G}, D)$. It follows that the corresponding closed point of $T_{p} G$ is a maximal D-ideal of $\left(\mathcal{O}_{\tilde{G}}, D\right)$ containing $X_{0}$, and hence it is in $U_{G}$. Conversely, we have $U_{G}(K) \subseteq T_{p} G(K)$.

Lemma 5.15. $U_{G}(K)=U_{G}(\bar{K})$.

Proof. Let $\mathcal{I}_{U}$ be the sheaf of D-ideals defining $U_{G}$ (in fact, we consider its sections on an affine open set). The reduced scheme $\left(U_{G}\right)_{\text {red }}$ is defined by $\sqrt{\mathcal{I}_{U}}$. It is well known that $\sqrt{\mathcal{I}_{U}}$ is the intersection of all the prime ideals containing $\mathcal{I}_{U}$. But, since $\mathcal{I}_{U}$ is a differential ideal, $\sqrt{\mathcal{I}_{U}}$ is also the intersection of all the prime D-ideals containing $\mathcal{I}_{U}$ (see [1], for example).

Now consider $M$, any maximal ideal of $\mathcal{O}_{\tilde{G}}$ containing $\mathcal{I}_{U}$. We want to show that $M$ is a D-ideal. Let $f$ be in $M$; it is in $\mathcal{O}_{G_{i}} \subseteq \mathcal{O}_{\tilde{G}}$ for some $i$. Let $j<p^{n}$ be some index. From the first remark, $\cap P \subseteq M$, where $P$ runs over the prime D-ideals of $\mathcal{O}_{\tilde{G}}$ containing $\mathcal{I}_{U}$. In particular, $\bigcap\left(P \cap \mathcal{O}_{G_{i+n}}\right) \subseteq M \cap \mathcal{O}_{G_{i+n}}$, but the first one is the ideal defining $\left(\pi_{i+n}\left(U_{G}\right)\right)_{\text {red }}$, a finite scheme (here, $\pi_{i+n}$ is the projection of $\tilde{G}$ onto $\left.G_{i+n}\right)$. It follows that $M \cap \mathcal{O}_{G_{i+n}}=P \cap \mathcal{O}_{G_{i+n}}$ for some D-ideal $P$ containing $\mathcal{I}_{U}$ (which may depend on $i$ and $n)$. But now we have $D_{j}(f)=F^{n *} \circ D_{j}^{\prime} \circ V_{n}^{*} \circ s^{n *}(f)$, with $s^{n *}(f) \in \mathcal{O}_{G_{i+n}}$ by definition and $D_{j}(f)$ as well, because $\mathcal{O}_{G_{i+n}}$ is stable under $F^{n *} \circ D_{j}^{\prime} \circ V_{n}^{*}$. As $P$ is a D-ideal, we have $D_{j}(f) \in\left(P \cap \mathcal{O}_{G_{i+n}}\right) \subseteq M$. That is, $M$ is a D-ideal.

Now we can conclude that, for any point $x \in U_{G}(\bar{K})$, the corresponding maximal ideal $M$ of $\mathcal{O}_{\tilde{G}}$ is a D-ideal; hence the residue field $K(x)$ is an algebraic D-extension of $K$. But we know that any algebraic D-extension of $K$ is trivial because $K$ is existentially closed (see [41], for example); hence $x \in U_{G}(K)$.

In order to deal with a big chunk of the nonreduced part of $T_{p} G$, we introduce the following morphism of group schemes $\tilde{F}: \tilde{G} \rightarrow \tilde{\tilde{G}}$, for $\tilde{F}$ and $\tilde{\tilde{G}}$ defined as follows (recall that $F^{i}$ is the Frobenius homomorphism $\left.G \rightarrow G^{\left(p^{i}\right)}\right)$ : 


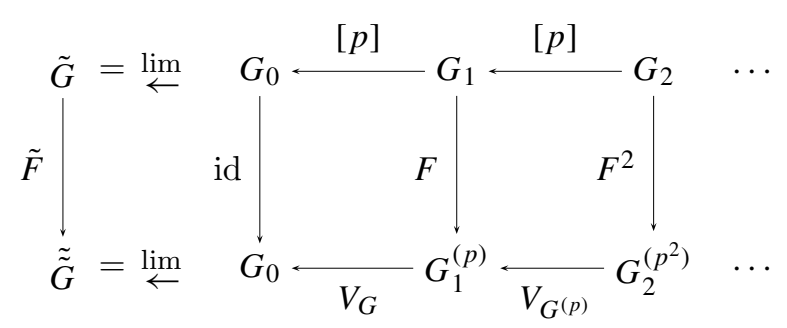

This is well defined since, for all $i, F^{i} \circ[p]=[p] \circ F^{i}=V_{G^{\left(p^{i}\right)}} \circ F^{i+1}$.

For each $i$, we will denote by $Y_{i}$ a system of coordinates of $G_{i}^{\left(p^{i}\right)}$ such that $F^{i *}\left(Y_{i}\right)=X_{i}^{p^{i}}$; $Y_{i}$ generates the maximal ideal of the identity element in $G_{i}^{\left(p^{i}\right)}$.

Lemma 5.16. $\operatorname{Ker}(\tilde{F})$ is a D-subscheme of $\tilde{G}$. In particular, $\operatorname{Ker}(\tilde{F}) \subseteq U_{G}$.

Proof. The sheaf of ideals $\mathcal{I}$ of the kernel is generated by the $F^{i *}\left(Y_{i}\right)$, that is, by the $X_{i}^{p^{i}}$. If $p^{i}$ does not divide $j, D_{j}\left(X_{i}^{p^{i}}\right)=0$; and if $j=h p^{i}$ for some $h<p^{n}$, we have $D_{j}\left(X_{i}^{p^{i}}\right)=\left(D_{h}\left(X_{i}\right)\right)^{p^{i}}$, with

$$
D_{h}\left(X_{i}\right)=F^{n *} \circ D_{h}^{\prime} \circ V_{n}^{*}\left(X_{i+n}\right) .
$$

Here, the map $V_{n}: \tilde{G}^{\left(p^{n}\right)} \rightarrow \tilde{G}$ comes from the homomorphisms $V_{n}: G_{k}^{\left(p^{n}\right)} \rightarrow G_{k}, k \geqslant 0$. It follows that $V_{n}^{*}\left(X_{i+n}\right) \in \mathcal{M}$, the maximal ideal of the identity element in $\mathcal{O}_{G_{i+n}^{\left(p^{n}\right)}}$. Since the

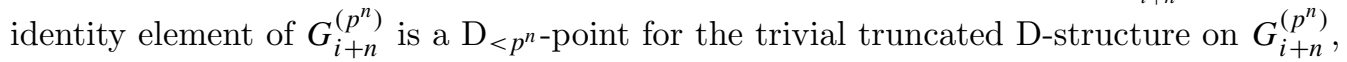
$D_{h}^{\prime}(\mathcal{M}) \subseteq \mathcal{M}$. Hence $D_{h}\left(X_{i}\right) \in\left(F^{n *}(\mathcal{M})\right) \subseteq\left(X_{i+n}^{p^{n}}\right)$, and $D_{j}\left(X_{i}^{p^{i}}\right) \in\left(X_{i+n}^{p^{i+n}}\right) \subseteq \mathcal{I}$.

We now need $G$ to be ordinary. We will use here the structure theorems for affine commutative group schemes over a field $L$ (note that $\operatorname{Ker}(\tilde{F}), U_{G}$, and $T_{p} G$ are objects of this category since they are commutative profinite), for which our reference is [13] (Chapter III, $\S 3$ and Chapter V, $\S 3$ ). Let us recall that, if $H$ is a profinite commutative group scheme over $L$, the connected component $H^{\circ}$ of the neutral element is an infinitesimal group subscheme, namely, it satisfies $H^{\circ}(\bar{L})=\{0\}$. Moreover, the quotient $H / H^{\circ}$ is proétale (that is, a projective limit of finite étale groups), and $H^{\circ}$ is the unique connected group subscheme of $H$ with this property. Furthermore, if we assume $L$ to be algebraically closed, the reduced subscheme $H_{\text {red }}$ is isomorphic to $H / H^{\circ}$ via the projection map (it is in particular a group subscheme of $H$ ), and $H$ is isomorphic to the direct product $H_{\text {red }} \times H^{\circ}$. Note finally that taking the connected component $H^{\circ}$ commutes with a base change of fields, and the same is true for taking the quotient $H / H^{\circ}$.

Lemma 5.17. Suppose that $G$ is ordinary. Then $\left(T_{p} G\right)^{\circ}=U_{G}^{\circ}=\operatorname{Ker}(\tilde{F})$.

Proof. We have seen that $\operatorname{Ker}(\tilde{F}) \subseteq U_{G} \subseteq T_{p} G$ (as closed group subschemes). But $T_{p} G / \operatorname{Ker}(\tilde{F})$ is a group subscheme of the projective limit

$$
\lim _{\leftarrow}\left(0 \stackrel{V_{G}}{\longleftarrow} \operatorname{Ker}\left(V_{1}\right) \stackrel{V_{G}(p)}{\longleftarrow} \operatorname{Ker}\left(V_{2}\right) \cdots\right),
$$


which is a proétale group scheme since each $\operatorname{Ker}\left(V_{n}\right)$ is étale $\left(V_{n}\right.$ is separable since $G$ is ordinary). It follows that $T_{p} G / \operatorname{Ker}(\tilde{F})$ and $U_{G} / \operatorname{Ker}(\tilde{F})$ are themselves proétale (this category is closed under subobjects). Since obviously $\operatorname{Ker}(\tilde{F})(\bar{K})=\{0\}$, the lemma is proved.

By combining the previous lemmas, we obtain a new proof of the Proposition 4.3 with a 'D-structure flavour'. Note that, for any profinite group scheme $X$ over $K, X_{\text {red }}$ is the unique reduced closed subscheme of $X$ such that $X(\bar{K})=X_{\text {red }}(\bar{K})$.

Proposition 5.18. Let $G$ be an ordinary semiabelian variety over $K$. Then $G$ descends to the constants if and only if $T_{p} G(\bar{K})=U_{G}(\bar{K})$ if and only if $T_{p} G(K)=T_{p} G(\bar{K})$.

Proof. We have obtained that $G$ descends to the constants if and only if $T_{p} G=U_{G}$ (5.13). But $T_{p} G=U_{G}$ if and only if $\left(T_{p} G\right)_{K}=\left(U_{G}\right)_{\bar{K}}$ (since field extensions are obviously faithfully flat), and, by the direct sum decomposition, $\left(T_{p} G\right)_{\bar{K}}=\left(U_{G}\right)_{\bar{K}}$ if and only if $\left(\left(T_{p} G\right)_{\bar{K}}\right)_{\mathrm{red}}=\left(\left(U_{G}\right)_{\bar{K}}\right)_{\mathrm{red}}$ and $\left(T_{p} G\right)_{\bar{K}}^{\circ}=\left(U_{G}\right)_{\bar{K}}^{\circ}$. But, by $5.17,\left(T_{p} G\right)^{\circ}=U_{G}^{\circ}$; hence $T_{p} G=U_{G}$ if and only if $T_{p} G(\bar{K})=U_{G}(\bar{K})$. Since $U_{G}(\bar{K})=U_{G}(K)=T_{p} G(K)(5.14$ and 5.15), we have the result.

\subsection{Uniform statements and proofs}

We will consider $G$ a semiabelian variety over $K$. Recall that, in characteristic $0, \tilde{G}$ is the universal extension of $G$ by a vector group, and that $W_{G}$ and $U_{G}$ are defined as described in Fact 5.7. In characteristic $p, \tilde{G}, U_{G}$, and $W_{G}=T_{p} G$ have been defined in $\S 5.2$.

In all characteristics, we know from Fact 5.7 in characteristic 0, and from Lemmas 4.2 and 5.14 in characteristic $p$, that

$$
G^{\sharp} \text { is isomorphic to }(\tilde{G}, \partial)^{\partial}(K) /\left(U_{G}, \partial\right)^{\partial}(K),
$$

where, of course, by isomorphic here we mean isomorphic as $*$-definable subgroups.

Notation. If $f: G \longrightarrow H$ is a morphism of semiabelian varieties over $K$, we denote by $\tilde{f}$ the induced morphism from $\tilde{G}$ to $\tilde{H}$.

In the following, we will often drop the $\partial$ for a scheme $(X, \partial)$ with a D-structure when no ambiguity arises.

If $H_{1}, H_{2}$ are proalgebraic groups over $K$ with a D-structure, and $h: H_{1} \longrightarrow H_{2}$ is a morphism of proalgebraic groups with a D-structure, we denote by $h^{\partial}$ the induced *-definable homomorphism from $H_{1}{ }^{\partial}(K)$ to $H_{2}{ }^{\partial}(K)$. When $G, H$ are semiabelian varieties, $\tilde{G}$ and $\tilde{H}$ have unique D-structures, and so, for any $f: G \rightarrow H, \tilde{f}$ respects the D-structures (see Remark 5.10 in characteristic p, and Corollary 3.6 from [6] in characteristic 0 ), whereby $\tilde{f}^{\partial}$ is defined. For the same reason, $\tilde{f}$ induces the maps $\tilde{f}_{U}: U_{G} \rightarrow U_{H}$ and $\left(\tilde{f}_{U}\right)^{\partial}: U_{G}^{\partial}(K) \rightarrow U_{H}^{\partial}(K)$ (see $\S \S 5.1$ and 5.2).

Lemma 5.19. Let $0 \longrightarrow G_{1} \longrightarrow G_{2} \stackrel{f}{\longrightarrow} G_{3} \longrightarrow 0$ be an exact sequence of semiabelian varieties over $K$. Then the sequence $0 \longrightarrow\left(\tilde{G}_{1}\right)^{\partial}(K) \longrightarrow\left(\tilde{G}_{2}\right)^{\partial}(K) \stackrel{\tilde{f}^{\partial}}{\longrightarrow}\left(\tilde{G}_{3}\right)^{\partial}(K) \longrightarrow 0$ is also exact. 
Proof. In characteristic $0, \tilde{G}_{i}$ is the universal vectorial extension of $G_{i}$, and the sequence

$$
0 \longrightarrow \tilde{G}_{1} \longrightarrow \tilde{G}_{2} \stackrel{\tilde{f}}{\longrightarrow} \tilde{G}_{3} \longrightarrow 0
$$

is also exact (see Appendix B). Each $\tilde{G}_{i}$ admits a (unique) D-structure, and the functor $H \mapsto H^{\partial}(K)$ preserves exact sequences from the category of algebraic groups with a D-structure to the category of definable groups (see [17]). In characteristic $p$, the sequence $0 \rightarrow G_{1}(K) \rightarrow G_{2}(K) \rightarrow G_{3}(K) \rightarrow 0$ is exact because $K$ is separably closed; hence, passing to the projective limit in the category of $*$-definable groups,

$$
0 \longrightarrow \tilde{G}_{1}(K) \longrightarrow \tilde{G}_{2}(K) \stackrel{\tilde{f}}{\longrightarrow} \tilde{G}_{3}(K)
$$

is also exact. The fact that $\tilde{f}: \tilde{G}_{2}(K) \rightarrow \tilde{G}_{3}(K)$ is surjective follows from the surjectivity of $f: G_{2}(K) \rightarrow G_{3}(K)$ and from the $\omega_{1}$-saturation of $K$.

The next proposition gives us a very useful equivalent to the exactness of the $\sharp$ functor. It should be noted that there is no assumption that any of the $U_{G_{i}}^{\partial}$, or any of the $U_{G_{i}}$, are nontrivial.

Given the exact sequence $0 \longrightarrow G_{1} \longrightarrow G_{2} \stackrel{f}{\longrightarrow} G_{3} \longrightarrow 0, \tilde{f},(\tilde{f})^{\partial}, \tilde{f}_{U}$, and $\left(\tilde{f}_{U}\right)^{\partial}$ are the induced maps as above, and $\tilde{f}_{\pi}$ denotes the induced map from $G_{2}^{\sharp}$ to $G_{3}^{\sharp}$, when we identify $G_{i}^{\sharp}$ with $\left(\tilde{G}_{i}\right)^{\partial}(K) /\left(U_{G_{i}}\right)^{\partial}(K)$.

Proposition 5.20. Let $0 \longrightarrow G_{1} \longrightarrow G_{2} \stackrel{f}{\longrightarrow} G_{3} \longrightarrow 0$ be an exact sequence of semiabelian varieties over $K$. For convenience, we assume that $G_{1}$ is a semiabelian subvariety of $G_{2}$. Then the following are equivalent.

(i) $0 \longrightarrow G_{1}^{\sharp} \longrightarrow G_{2}^{\sharp} \stackrel{f_{\pi}}{\longrightarrow} G_{3}^{\sharp} \longrightarrow 0$ is exact.

(ii) $0 \longrightarrow\left(U_{G_{1}}\right)^{\partial}(K) \longrightarrow\left(U_{G_{2}}\right)^{\partial}(K) \stackrel{\left(\tilde{f}_{U}\right)^{\partial}}{\longrightarrow}\left(U_{G_{3}}\right)^{\partial}(K) \longrightarrow 0$ is exact.

(iii) $\left(\tilde{f}_{U}\right)^{\partial}:\left(U_{G_{2}}\right)^{\partial}(K) \longrightarrow\left(U_{G_{3}}\right)^{\partial}(K)$ is surjective.

(iv) $0 \longrightarrow U_{G_{1}}(\bar{K}) \longrightarrow U_{G_{2}}(\bar{K}) \stackrel{\tilde{f}_{U}}{\longrightarrow} U_{G_{3}}(\bar{K}) \longrightarrow 0$ is exact.

Furthermore, $\left(G_{1}(K) \cap G_{2}^{\sharp}\right) / G_{1} \sharp \widetilde{\longrightarrow}\left(U_{G_{3}}\right)^{\partial}(K) /\left(\tilde{f}_{U}\right)^{\partial}\left(\left(U_{G_{2}}\right)^{\partial}(K)\right)$.

Proof. From Lemma 5.19, one obtains the following commutative diagram of exact sequences $(*)$ :

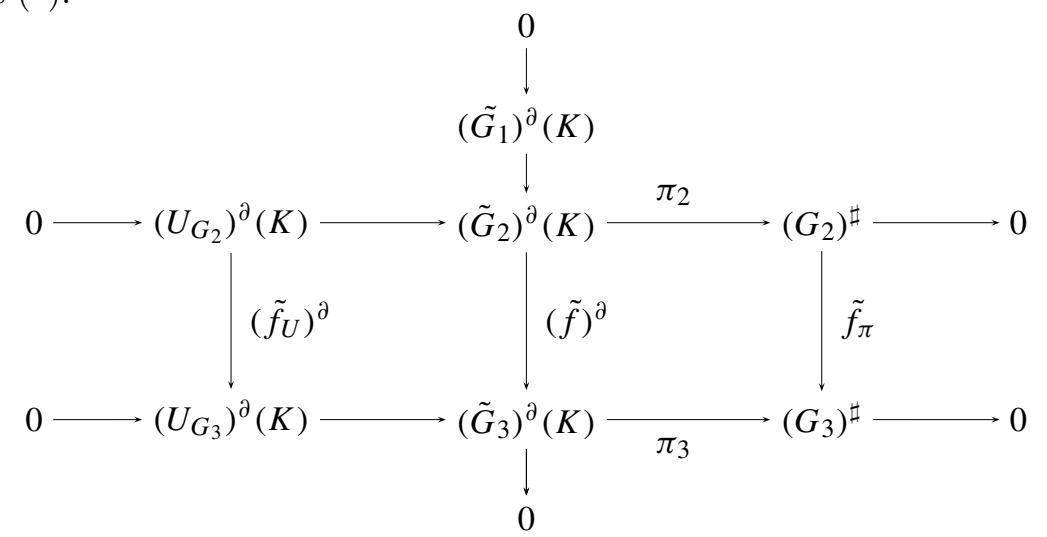


Claim. $\operatorname{Ker}\left(\tilde{f}_{U}\right)^{\partial}=\left(U_{G_{1}}\right)^{\partial}(K)$.

First, note that $\left(U_{G_{i}}\right)^{\partial}(K)=\left(\tilde{G}_{i}\right)^{\partial}(K) \cap W_{i}$, since it is the kernel of the restriction of $\pi_{i}$ to $\left(\tilde{G}_{i}\right)^{\partial}(K)$, and $W_{2} \cap \tilde{G}_{1}=W_{1}$, since $W_{i}$ is the kernel of $\pi_{i}: \tilde{G}_{i} \rightarrow G_{i}$. It follows that $\left(U_{G_{1}}\right)^{\partial}(K)=\left(\tilde{G}_{1}\right)^{\partial}(K) \cap W_{1}=\left(\tilde{G}_{1}\right)^{\partial}(K) \cap W_{2}=\left(\tilde{G}_{1}\right)^{\partial}(K) \cap\left(\tilde{G}_{2}\right)^{\partial}(K) \cap$ $W_{2}=\operatorname{Ker}\left((\tilde{f})^{\partial}\right) \cap\left(U_{G_{2}}\right)^{\partial}(K)=\operatorname{Ker}\left(\tilde{f}_{U}\right)^{\partial}$.

Let $S:=\left(U_{G_{3}}\right)^{\partial}(K) /\left(\tilde{f}_{U}\right)^{\partial}\left(\left(U_{G_{2}}\right)^{\partial}(K)\right)$ (the cokernel of $\left.\left(\tilde{f}_{U}\right)^{\partial}\right)$. Then the classical Snake Lemma applied to diagram $(*)$ gives the existence of a homomorphism $d$ from $\operatorname{Ker}\left(\tilde{f}_{\pi}\right)$ to $S$, such that the sequence $0 \longrightarrow\left(U_{G_{1}}\right)^{\partial} \longrightarrow\left(\tilde{G}_{1}\right)^{\partial} \longrightarrow \operatorname{Ker}\left(\tilde{f}_{\pi}\right) \stackrel{d}{\longrightarrow} S \longrightarrow 0 \longrightarrow 0$ is exact in the following commutative diagram:

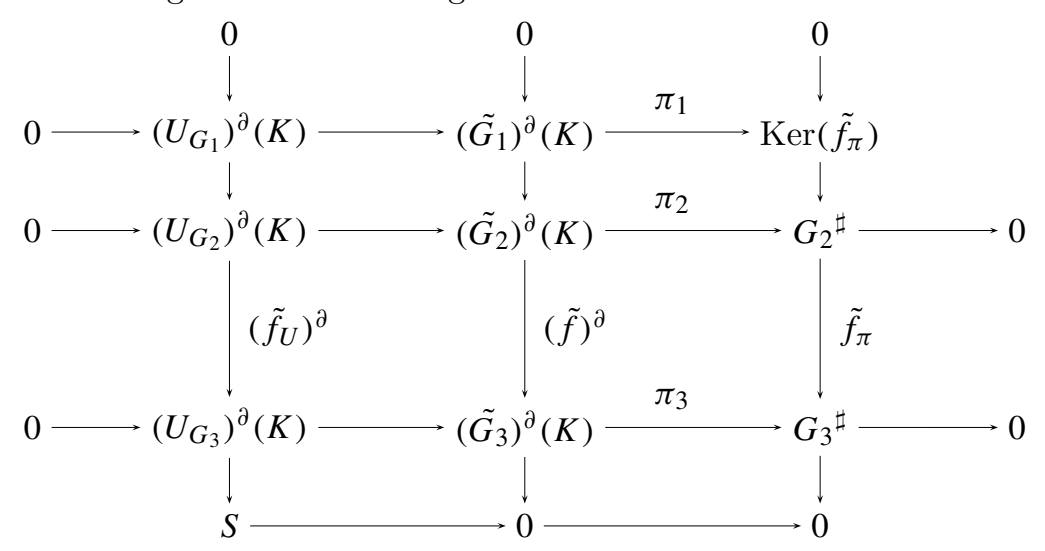

This says exactly that

$S=\left(U_{G_{3}}\right)^{\partial}(K) /\left(\tilde{f}_{U}\right)^{\partial}\left(\left(U_{G_{2}}\right)^{\partial}(K)\right)$ is isomorphic to $\operatorname{Ker}\left(\tilde{f}_{\pi}\right) / \pi_{1}\left(\left(\tilde{G}_{1}\right)^{\partial}(K) /\left(U_{G_{1}}\right)^{\partial}(K)\right)$, that is, to $\left(G_{1}(K) \cap G_{2}^{\sharp}\right) / G_{1}^{\sharp}$.

It follows in particular that

$0 \longrightarrow G_{1}^{\sharp} \longrightarrow G_{2} \stackrel{f_{\pi}}{\longrightarrow} G_{3}{ }^{\sharp} \longrightarrow 0$ is exact

if and only if

$0 \longrightarrow\left(U_{G_{1}}\right)^{\partial}(K) \longrightarrow\left(U_{G_{2}}\right)^{\partial}(K) \stackrel{\left(\tilde{f}_{U}\right)^{\partial}}{\longrightarrow}\left(U_{G_{3}}\right)^{\partial}(K) \longrightarrow 0$ is exact

if and only if $\left(\tilde{f}_{U}\right)^{\partial}$ is surjective.

This is also equivalent to the exactness of the sequence $0 \longrightarrow U_{G_{1}}(\bar{K}) \longrightarrow U_{G_{2}}(\bar{K}) \stackrel{\left(\tilde{f}_{U}\right)}{\longrightarrow}$ $U_{G_{3}}(\bar{K}) \longrightarrow 0$. In characteristic 0 , one direction follows from the exactness of the $\partial$ functor on groups with a D-structure. For the other direction, suppose that the sequence of the $\left(U_{G_{i}}\right)^{\partial}(K)$ is exact. For each $i, U_{G_{i}}^{\partial}(K)$ has transcendence degree equal to the dimension of the algebraic group $U_{G_{i}}$ (Fact 5.3). It follows that $\operatorname{dim} U_{G_{1}}+\operatorname{dim} U_{G_{3}}=$ $\operatorname{dim} U_{G_{2}}$ (by additivity of the transcendence degree). Being vector groups, the sequence of the $U_{G_{i}}$ is exact. In characteristic $p$, this is a direct consequence of Lemmas 5.14 and 5.15 .

We can now give a uniform proof of the main result which relates exactness of the $\sharp$ functor to questions of descent, restricted, in char. $p$ to the class of ordinary semiabelian varieties. It is the uniform version of Proposition 4.12. 
Proposition 5.21. Let $0 \rightarrow G_{1} \rightarrow G_{2} \rightarrow G_{3} \rightarrow 0$ be an exact sequence of (ordinary in char. p) semiabelian varieties defined over $K$. Suppose that $G_{1}$ and $G_{3}$ descend to the constants of $K$.

Then the sequence $0 \rightarrow G_{1}^{\sharp} \rightarrow G_{2}^{\sharp} \rightarrow G_{3}^{\sharp} \rightarrow 0$ remains exact if and only if $G_{2}$ also descends to the constants.

Proof. Without loss of generality, we can suppose that $G_{1}$ is a semiabelian subvariety of $G_{2}$, and that $G_{1}=\left(G_{1}^{\prime}\right)_{K}$ and $G_{3}=\left(G_{3}^{\prime}\right)_{K}$, where $G_{1}^{\prime}$ and $G_{3}^{\prime}$ are semiabelian varieties over $\mathcal{C}$, the field of constants of $K$.

If $G_{2}$ descends to the constants, then, up to isomorphism, we can suppose that $G_{2}=$ $\left(G_{2}^{\prime}\right)_{K}$ for some $G_{2}^{\prime}$ over the constants, so, for every $i, G_{i}^{\sharp}=G_{i}^{\prime}(\mathcal{C})$. And then $G_{1}(K) \cap$ $G_{2}^{\sharp}=G_{1}(K) \cap G_{2}(\mathcal{C})=G_{1}(\mathcal{C})=G_{1}^{\sharp}$.

For the converse, suppose that $0 \rightarrow G_{1}^{\sharp}(K) \rightarrow G_{2}^{\sharp}(K) \rightarrow G_{3}^{\sharp}(K) \rightarrow 0$ is exact.

By Proposition 5.20, $0 \rightarrow U_{G_{1}}(\bar{K}) \rightarrow U_{G_{2}}(\bar{K}) \rightarrow U_{G_{3}}(\bar{K}) \rightarrow 0$ is also exact. We know that (see Fact 5.7 in characteristic 0 and Proposition 5.18 in characteristic $p$ ), as $G_{1}$ and $G_{3}$ descend to the constants, $U_{G_{1}}(\bar{K})=W_{1}(\bar{K})$ and $U_{G_{3}}(\bar{K})=W_{3}(\bar{K})$. Consider the dimensions, as vector spaces in characteristic 0 or as free $\mathbb{Z}_{p}$-modules in characteristic $p$, of the $U_{G_{i}}(\bar{K})$. By exactness, $\operatorname{dim}\left(U_{G_{2}}(\bar{K})\right)=\operatorname{dim}\left(U_{G_{1}}(\bar{K})\right)+\operatorname{dim}\left(U_{G_{3}}(\bar{K})\right)$. But we also have that $\operatorname{dim} W_{2}(\bar{K})=\operatorname{dim} W_{1}(\bar{K})+\operatorname{dim} W_{3}(\bar{K})$ (this follows from exactness of the functor $G \mapsto \tilde{G}$, which is clear in characteristic $p$, and proved in Appendix B for characteristic 0$)$. So $\operatorname{dim} U_{G_{2}}(\bar{K})=\operatorname{dim} W_{2}(\bar{K})$, and hence $U_{G_{2}}(\bar{K})=W_{2}(\bar{K})$; that is, again by Fact 5.7 in characteristic 0 and Proposition 5.18 in characteristic $p, G_{2}$ descends to the constants.

Hence we obtain in arbitrary characteristic the analogue of Corollary 4.13, with the same proof.

Corollary 5.22. For any ordinary abelian variety $A$ defined over the constants of $K$, there exists an exact sequence over $K$,

$$
0 \longrightarrow \mathbb{G}_{m} \longrightarrow H \longrightarrow A_{K} \longrightarrow 0
$$

such that

$$
0 \longrightarrow \mathbb{G}_{m}^{\sharp} \longrightarrow H^{\sharp} \longrightarrow\left(A_{K}\right)^{\sharp} \longrightarrow 0
$$

is not exact.

We have given some examples of nonexactness in characteristic $p$ in $\S 4.3$, even for abelian varieties. In characteristic 0, the situation is completely different for abelian varieties, as shown in the next Proposition, which is a direct consequence of Proposition 5.20.

Proposition 5.23 (Characteristic 0). Let $0 \longrightarrow A \longrightarrow B \longrightarrow C \longrightarrow 0$ be an exact sequence of abelian varieties over $K$. Then the induced sequence $0 \longrightarrow A^{\sharp} \longrightarrow B^{\sharp} \longrightarrow$ $C^{\sharp} \longrightarrow 0$ is also exact.

Proof. By Poincaré complete reducibility, $A \times C$ is isogenous to $B$, inducing an isogeny of $\widehat{A \times C}=\tilde{A} \times \tilde{C}$ with $\tilde{B}$. As this is also an isogeny of D-groups, it induces an isogeny 
between $U_{A \times C}=U_{A} \times U_{C}$ and $U_{B}$. As these are vector groups, it follows that the induced sequence $0 \longrightarrow U_{A} \longrightarrow U_{B} \longrightarrow U_{C} \longrightarrow 0$ is exact. Hence, by Proposition 5.20 , so is $0 \longrightarrow A^{\sharp} \longrightarrow B^{\sharp} \longrightarrow C^{\sharp} \longrightarrow 0$.

\section{Additional remarks and questions}

(1) In characteristic $p$, the counterexamples to exactness of the induced $\sharp$ sequence arise from the following situation: we have two connected commutative definable groups $G_{1}<G_{2}$ which are not divisible. We consider $D_{2}$, the biggest divisible subgroup (which is infinitely definable) of $G_{2}$. The counterexamples are exactly the cases when $G_{1} \cap D_{2}$ is not divisible. One can ask the same question also for other classes of groups, in particular for commutative algebraic groups. Given $G_{1}<G_{2}$ two commutative connected algebraic groups defined over some algebraically closed field $K$ of characteristic $p$, consider $D<G_{2}$, the biggest divisible subgroup of $G_{2}$. It is easy to check that $D$ is a closed subgroup of $G_{2}$, also defined over $K$.

Using the characterizations of the groups $p^{\infty} G(K)$, given in terms of the Weil restrictions $\Pi_{K / K^{p^{n}}} G$ in [4], one can deduce easily from our examples that the same phenomenon occurs for commutative algebraic groups.

(2) In previous drafts of this paper, we had mentioned an open question which we found quite intriguing. Let $A$ be an abelian variety defined over $\mathbb{F}_{p}(t)$, and let $K_{0}$ denote the separable closure of $\mathbb{F}_{p}(t)$. We can consider $A\left(K_{0}\right)$ and $p^{\infty} A\left(K_{0}\right)$. As we recalled in $\S 3, p^{\infty} A\left(K_{0}\right)$ is the biggest divisible subgroup of $A\left(K_{0}\right)$, and it contains all the torsion of $A$ which is prime to $p$. The question was whether $p^{\infty} A\left(K_{0}\right)$ could contain any nontorsion element. Note that, if $A$ is defined over $K_{0} p^{\infty}=\overline{\mathbb{F}_{p}}$, then $p^{\infty} A\left(K_{0}\right)=A\left(\overline{\mathbb{F}_{p}}\right)$, where indeed every element is torsion. Note also that, from the beginning of $\S 3$, in characteristic $p$, when dealing with $A^{\sharp}=p^{\infty} A(K)$, we suppose that $K$ is $\omega_{1}$-saturated, which ensures that $A^{\sharp}$ contains elements which are not torsion. This question was answered in some particular cases in [2], and in full generality by D. Rössler, who showed in [32] that $p^{\infty} A\left(K_{0}\right)$ contains only torsion points.

In characteristic 0 there are results along these lines, sometimes going under the expression 'Manin's theorem of the kernel'. A formal statement and proof (depending on results of Manin, Chai and others) appears in [6] (Corollary K.3 of the Appendix), and says that, if $A$ is an abelian variety over the algebraic closure $K_{0}$ say of $\mathbb{C}(t)$, equipped with a derivation with field of constants $\mathbb{C}$, and $A$ has $\mathbb{C}$-trace 0 , then $A^{\sharp}\left(K_{0}\right)$ is precisely the group of torsion points of $A$. This, together with the fact (see [6], $\S 6$ and [22], Lemma 2.2) that $A^{\sharp}\left(K_{0}\right)=A^{\sharp}\left(K_{0}^{\text {diff }}\right)$, shows that $A^{\sharp}\left(K_{0}{ }^{\operatorname{diff}}\right)$ is the group of torsion points of $A$.

\section{A. Appendix}

Here is a proof of Fact 2.3. Let $G$ and $H$ be two connected algebraic groups defined over $K$, and let $f$ be a dominant separable homomorphism from $G$ to $H$ (equivalently a surjective separable homomorphism from $G(\bar{K})$ to $H(\bar{K})$ ). Then $f$ takes $G(K)$ surjectively onto $H(K)$. 
Proof. Note first that we can suppose without loss of generality that $K$ is sufficiently saturated. Let $K_{0}$ be a small field over which everything is defined. Let $h \in H(K)$ be a generic point of $H$ over $K_{0}$ (in the sense of algebraic geometry). As $f$ is dominant, there is some generic $g$ of $G(\bar{K})$ such that $f(g)=h$. Separability of $f$ means that $K_{0}(g)$ is a separable extension of $K_{0}(h)$, and hence is contained in a separable closure of $K_{0}(h)\left(a_{1}, \ldots, a_{n}\right)$ for some $a_{i}$ which are algebraically independent over $K_{0}(h)$. Choosing, by saturation of $K, b_{1}, \ldots, b_{n} \in K$, algebraically independent over $K_{0}(h)$, and an isomorphism taking the separable closure of $K_{0}(h)\left(a_{1}, \ldots, a_{n}\right)$ to the separable closure of $K_{0}(h)\left(b_{1}, \ldots, b_{n}\right)$, we find $g^{\prime} \in G(K)$ such that $f\left(g^{\prime}\right)=h$.

\section{B. Appendix}

In this appendix, we give a detailed proof of the fact used in Lemma 5.19, namely that the functor 'universal extension', on the category of semiabelian varieties in characteristic 0 , is exact. As we could not find any references for this fact, which is possibly well known, we give the details here, thanks to the help of D. Bertrand. We refer to [5] for discussion about related questions. Note also that the point of view of rigidified extensions used in [23] should give this result more directly, but we keep here a point of view which model theorists are probably more familiar with.

Everything here is over an algebraically closed field $K$ of characteristic 0 , and every algebraic group is commutative.

Recall that the universal extension of an algebraic group $A$ by a vector group (when it exists) is an extension

$$
0 \rightarrow W_{A} \rightarrow \tilde{A} \stackrel{\pi_{A}}{\rightarrow} A \rightarrow 0
$$

where $W_{A}$ is a vector group, characterized by the following universal property: for every extension $f: G \rightarrow A$ of $A$ by a vector group, there exists a unique homomorphism of algebraic groups $g: \tilde{A} \rightarrow G$ such that $\pi_{A}=f \circ g$.

It follows from [31] that abelian varieties admit such universal extension. If $S$ is a semiabelian variety, with abelian part $A, S$ admits a universal extension by a vector group, which is given by $\tilde{S}=S \times_{A} \tilde{A}$ (see [6]); note that $W_{S}=W_{A}$.

We should now explain how ${ }^{\sim}$ is defined as a functor on the category of semiabelian varieties.

First, recall some notation and constructions from [34], Chapter 7. For algebraic groups $A$ and $B, \operatorname{Ext}(A, B)$ is the set of extensions $0 \rightarrow B \rightarrow C \rightarrow A \rightarrow 0$ of $A$ by $B$, up to isomorphism of extensions. It is equipped with a structure of a group.

If $C \in \operatorname{Ext}(A, B)$, and $g: B \rightarrow B^{\prime}, g_{*}(C)$ is the unique element $C^{\prime} \in \operatorname{Ext}\left(A, B^{\prime}\right)$ such that there is some $G: C \rightarrow C^{\prime}$ such that the following diagram commutes (actually it does not depend only on $C$, but on $C$ as an extension of $A$ by $B$ ):

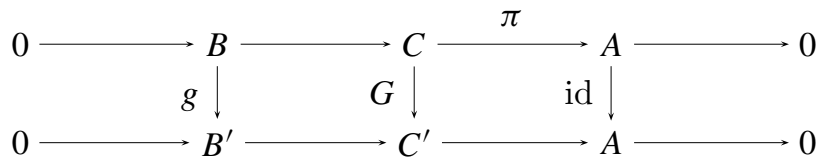


Note that such a $G$ does not need to be unique. By diagram chasing, we see that $G^{\prime}$ : $C \rightarrow C^{\prime}$ satisfies the same property as $G$ if and only if it can be written as $G^{\prime}=G+\Delta \circ \pi$ for some $\Delta \in \operatorname{Hom}\left(A, B^{\prime}\right)$.

Similarly, if $C \in \operatorname{Ext}(A, B)$, and $f: A^{\prime} \rightarrow A, f^{*}(C)$ is the unique element $C^{\prime} \in$ $\operatorname{Ext}\left(A^{\prime}, B\right)$ such that there is some $F: C^{\prime} \rightarrow C$ such that the following diagram commutes:

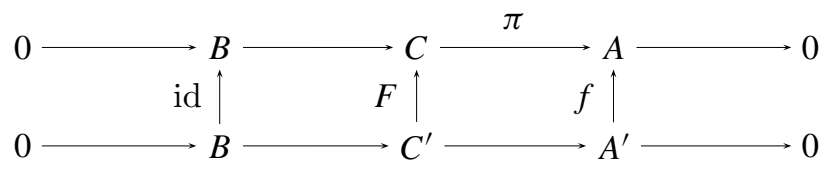

As before, $F^{\prime}: C^{\prime} \rightarrow C$ satisfies the same property as $F$ if and only if $F^{\prime}=F+\Delta \circ \pi$ for some $\Delta \in \operatorname{Hom}\left(A^{\prime}, B\right)$.

We can give an explicit description of $f^{*}(C)$ : it is (isomorphic to) $C \times{ }_{A} A^{\prime}$, viewed as an extension of $A^{\prime}$ via the second projection, and with map to $C$ given by the first projection.

An important result is Proposition 2 of Chapter 7 in [34]. An exact sequence $0 \rightarrow$ $A_{1} \stackrel{f}{\rightarrow} A_{2} \stackrel{g}{\rightarrow} A_{3} \rightarrow 0$ and an algebraic group $H$ induce an exact sequence $\left(^{*}\right)$ :

$$
\begin{aligned}
0 & \rightarrow \operatorname{Hom}\left(A_{3}, H\right) \stackrel{\circ g}{\rightarrow} \operatorname{Hom}\left(A_{2}, H\right) \stackrel{\circ \circ}{\rightarrow} \operatorname{Hom}\left(A_{1}, H\right) \stackrel{d}{\rightarrow} \operatorname{Ext}\left(A_{3}, H\right) \\
& \stackrel{g^{*}}{\rightarrow} \operatorname{Ext}\left(A_{2}, H\right) \stackrel{f^{*}}{\rightarrow} \operatorname{Ext}\left(A_{1}, H\right),
\end{aligned}
$$

where $d(\phi)=\phi_{*}\left(A_{2}\right) \in \operatorname{Ext}\left(A_{3}, H\right)$ for $\phi \in \operatorname{Hom}\left(A_{1}, H\right)$.

Note that in the following situation

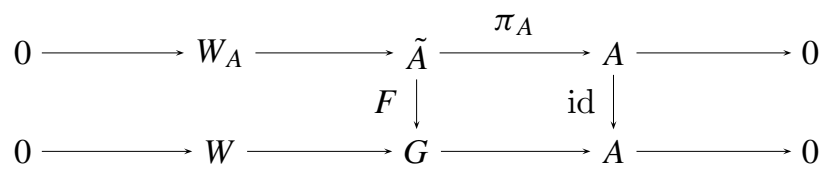

where $A$ is a semiabelian variety, $G$ an extension of $A$ by a vector group $W$, and $F$ given by the universal property, $F$ must map the unipotent part $W_{A}$ of $\tilde{A}$ into the unipotent part $W$ of $G$. Hence, by definition, the restriction $F_{W}: W_{A} \rightarrow W$ is such that $G=\left(F_{W}\right)_{*}(\tilde{A})$. Furthermore, since $\operatorname{Hom}(A, W)=0, F_{W}$ completely determines $F$. Hence finding $F$ as in the universal property is equivalent to finding the unique $f: W_{A} \rightarrow W$ such that $f_{*}(\tilde{A})=G$.

We will now use this characterization in order to build $\tilde{f}: \tilde{A} \rightarrow \tilde{B}$ for $f: A \rightarrow$ $B$ an homomorphism of semiabelian varieties. For such an $f$, define $T f$ as the unique $T f: W_{A} \rightarrow W_{B}$ such that $(T f)_{*}(\tilde{A})=f^{*}(\tilde{B})$. Because of the definitions, we get homomorphisms $G: \tilde{A} \rightarrow(T f)_{*}(\tilde{A})$ and $F: f^{*}(\tilde{B}) \rightarrow \tilde{B}$ making the following diagram commutative:

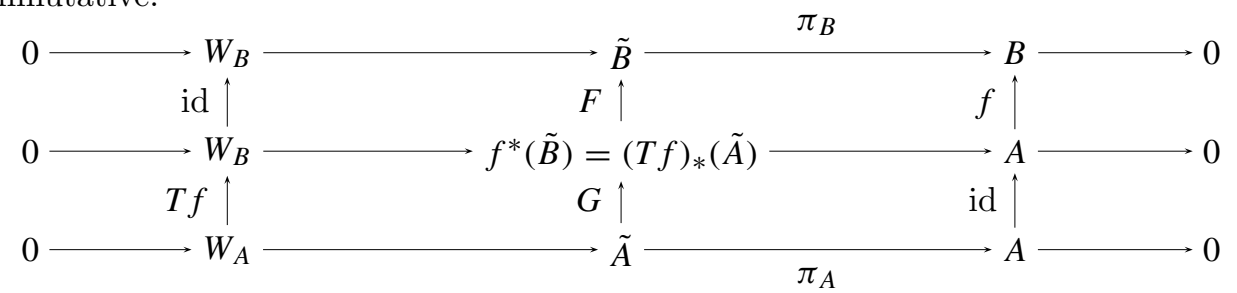


Now we define $\tilde{f}=F \circ G$; it makes the following diagram commutative:

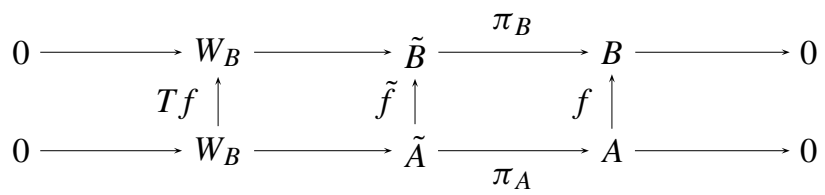

and it is the unique such (once again using that $\operatorname{Hom}\left(A, W_{B}\right)=0$ ).

With these characterizations, it is easy to show that, for homomorphisms of semiabelian varieties $A \stackrel{f}{\rightarrow} B \stackrel{g}{\rightarrow} C, \tilde{g f}=\tilde{g} \tilde{f}$ : the calculation $(g f)^{*}(\tilde{C})=f^{*} g^{*}(\tilde{C})=f^{*}(T g)_{*}(\tilde{B})=$ $(T g)_{*} f^{*}(\tilde{B})=(T g)_{*}(T f)_{*}(\tilde{A})=(T g T f)_{*}(\tilde{A})$ shows that $T(g f)=T g T f$, and the result follows (the basic results that we use here about $f^{*}$ and $g_{*}$ can be found in [31] or [34]).

Now we prove exactness.

We will use the natural identification of $W_{A}$ with the dual of $\operatorname{Ext}\left(A, \mathbb{G}_{a}\right)$. More precisely, if $A$ is an abelian variety, the map

$$
\begin{aligned}
\operatorname{Hom}\left(W_{A}, \mathbb{G}_{a}\right) & \rightarrow \operatorname{Ext}\left(A, \mathbb{G}_{a}\right) \\
\phi & \mapsto \phi_{*} \tilde{A}
\end{aligned}
$$

is an isomorphism (see [31], Prop. 11).

The same result is valid for a semiabelian variety $0 \rightarrow T \rightarrow S \stackrel{f}{\rightarrow} A \rightarrow 0$ instead of $A$. Indeed, since $\operatorname{Hom}\left(T, \mathbb{G}_{a}\right)=\operatorname{Ext}\left(T, \mathbb{G}_{a}\right)=0$, it follows from the exact sequence $(*)$ that $f^{*}: \operatorname{Ext}\left(A, \mathbb{G}_{a}\right) \rightarrow \operatorname{Ext}\left(S, \mathbb{G}_{a}\right)$ is an isomorphism. But, by construction, $\tilde{S}=f^{*} \tilde{A} \in$ $\operatorname{Ext}\left(S, W_{A}\right)$, and, for $\phi \in \operatorname{Hom}\left(W_{A}, \mathbb{G}_{a}\right), \phi_{*} \tilde{S}=\phi_{*} f^{*} \tilde{A}=f^{*} \phi_{*} \tilde{A}$; hence the result comes from the case of abelian varieties.

Claim B.1. For $f: A \rightarrow B$ an homomorphism of semiabelian varieties, the following diagram commutes:

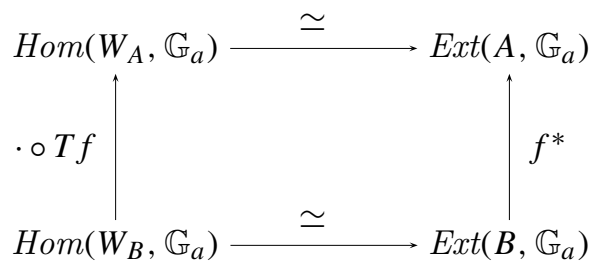

Indeed, for $\phi \in \operatorname{Hom}\left(W_{B}, \mathbb{G}_{a}\right),(\phi \circ T f)_{*} \tilde{A}=\phi_{*}(T f)_{*} \tilde{A}=\phi_{*} f^{*} \tilde{B}=f^{*} \phi_{*} \tilde{B}$.

Now we consider an exact sequence of semiabelian varieties

$$
0 \rightarrow A \stackrel{f}{\rightarrow} B \stackrel{g}{\rightarrow} C \rightarrow 0
$$

Claim B.2. The induced sequence is exact:

$$
0 \rightarrow \operatorname{Ext}\left(C, \mathbb{G}_{a}\right) \stackrel{g^{*}}{\rightarrow} \operatorname{Ext}\left(B, \mathbb{G}_{a}\right) \stackrel{f^{*}}{\rightarrow} \operatorname{Ext}\left(A, \mathbb{G}_{a}\right) \rightarrow 0 .
$$


We use the exact sequence $\left(^{*}\right)$ and the fact that $\operatorname{Hom}\left(A, \mathbb{G}_{a}\right)=0$ to get the exactness on the left and on the middle. For the surjectivity, we just have to use the dimensions and connectedness of these groups, since the dimension of $\operatorname{Ext}\left(A, \mathbb{G}_{a}\right)$ equals the dimension of the abelian part of $A$.

Proposition B.3. The induced sequence is exact:

$$
0 \rightarrow \tilde{A} \stackrel{\tilde{f}}{\rightarrow} \tilde{B} \stackrel{\tilde{g}}{\rightarrow} \tilde{C} \rightarrow 0
$$

Proof. In the following commutative diagram

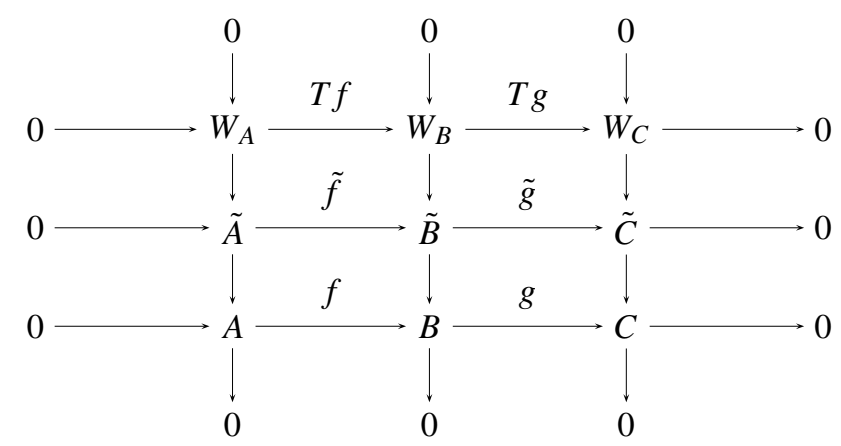

the columns and the bottom row are exact. The top row is exact by the two claims and duality. It follows that the middle row is exact.

Acknowledgements. Elisabeth Bouscaren would like to thank particularly Ehud Hrushovski and Françoise Delon for numerous discussions in the past years on the questions addressed in this paper. Grateful thanks from all three authors go especially to Daniel Bertrand and Damian Rössler for numerous and enlightening discussions. Among the many others who have helped with explanations or discussions with some of the authors, let us give special thanks to Jean-Benoit Bost, Antoine Chambert-Loir, Marc Hindry, Minhyong Kim, and Thomas Scanlon. Finally, we thank the referees of earlier versions for their comments, and particular thanks are given to the final referee for his/her careful reading and numerous extremely helpful remarks and suggestions.

\section{References}

1. F. Benoist, Théorie des modèles des corps munis d'une dérivation de Hasse, PhD thesis, Univ. Paris 7 (2005).

2. F. Benoist, A theorem of the Kernel in characteristic $p$, preprint, 2011.

3. F. Benoist, Schemes with D-structure, preprint, 2014, http://www.math.u-psud.fr/ fbenoist/Dstructure.pdf.

4. F. Benoist and F. Delon, Questions de corps de définition pour les variétés abéliennes en caractéristique positive, Journal de l'Institut de Mathématiques de Jussieu 7 (2008), 623-639.

5. D. Bertrand, Endomorphismes de groupes algébriques, in Diophantine Approximations and Transcendental Numbers (Luminy, 1982). 
6. D. Bertrand And A. Pillay, A Lindemann-Weierstrass theorem for semiabelian varieties over function fields, J. Amer. Math. Soc. 23 (2010), 491-533.

7. E. Bouscaren and F. Delon, Groups definable in separably closed fields, Trans. Amer. Math. Soc. 354 (2002), 945-966.

8. E. Bouscaren and F. Delon, Minimal groups in separably closed fields, J. Symbolic Logic 67 (2002), 239-259.

9. A. Bonel, Linear algebraic groups,2nd enlarged ed., Graduate Text in Mathematics, (Springer, New York, 1991).

10. A. Buium, Differential algebraic group of finite dimension, Lecture Notes in Mathematics 1506 (Springer-Verlag, 1992).

11. A. Buium, Differential Algebra and Diophantine Geometry (Hermann, Paris, 1994).

12. B. ConraD, Chow's $K / k$-image and $K / k$-trace and the Lang-Néron theorem, Enseign. Math. (2) 52 (2006), 37-108.

13. M. Demazure and P. Gabriel, Groupes algébriques Tome I (Masson, Paris, 1970).

14. M. M. Erimbetov, Complete theories with 1-cardinal formulas, Algebra Logika 14 (1975), $245-257$.

15. A. Grothendieck and M. Raynaud, Revêtements étales et groupe fondamental, in Séminaire de géométrie algébrique du Bois Marie (SGA1), 1960-61, Lecture Notes in Mathematics, Volume 224 (Springer-Verlag, 1971).

16. E. Hrushovski, The Mordell-Lang conjecture for function fields, J. Amer. Math. Soc. 9 (1996), 667-690.

17. P. Kowalski And A. PIllay, Quantifier elimination for algebraic $D$-groups, Trans. Amer. Math. Soc. 358 (2006), 167-181.

18. P. Kowalski AND A. Pillay, On the isotriviality of projective iterative $\partial$-varieties, J. Pure Appl. Algebra 216 (2012), 20-37.

19. S. LANG, Abelian Varieties (Interscience, London, 1959).

20. D. MARKER, Manin kernels, Quaderni Math., Volume 6, pp. 1-21 (Napoli, 2000).

21. D. MARKer, Model theory of differential fields, in Model Theory of Fields, second edition, Lecture Notes in Logic (ASL, AK Peters, 2006).

22. D. Marker and A. Pillay, Differential Galois Theory III: Some inverse problems, Illinois J. Math. 41 (1997), 453-461.

23. B. MAZUR AND W. Messing, Universal extensions and one dimensional crystalline cohomology, Lecture Notes in Mathematics, Volume 370 (Springer, 1974).

24. J. S. Milne, Etale cohomology (Princeton University Press, 1980).

25. R. Moosa And T. Scanlon, Jet and prolongations spaces, J. Inst. Math. Jussieu 9 (2010), 391-430.

26. D. Mumford, Abelian varieties (Oxford University Press, 1985). Published for the Tata Institute of Fundamental Research, Bombay.

27. D. Mumford And J. Fogarty, Geometric invariant theory, 2nd enlarged edition (Springer, 1982).

28. A. Pillay, Differential algebraic groups and the number of countable differentially closed fields, in Model Theory of Fields, cited above.

29. B. Poizat, Stable groups, Mathematical Surveys and Monographs (American Mathematical Society, 2001).

30. M. Rosenlicht, Some basic theorems on algebraic groups, Amer. J. Math 76 (1956), 401-443.

31. M. Rosenlicht, Extensions of vector groups by abelian varieties, Amer. J. Math. 80 (1958), 685-714. 
32. D. RössLeR, Infinitely $p$-divisible points on abelian varieties defined over function fields of characteristic $p>0$, Notre Dame J. Formal Logic 54 (2013), 579-589.

33. J.-P. SERre, Quelques propriétes des variétes abéliennes en caractéristique p, Amer. J. Math. 80(3) (1958), 715-739.

34. J.-P. SERRE, Algebraic groups and class fields, Graduate Texts in Mathematics (Springer, 1988).

35. S. Shelah, Classification Theory, 2nd ed. (North Holland, 1990).

36. J. H. Silverman, The arithmetic of elliptic curves, Graduate Texts in Mathematics (Springer-Verlag, 1986).

37. P. Vojta, Jets via Hasse-Schmidt derivations, in Diophantine Geometry, CRM Series, Volume 4, pp. 335-361 (Ed. Norm., Pisa, 2007).

38. F. Voloch, Diophantine approximation on Abelian varieties in characteristic p, Amer. J. Math. 4 (1995), 1089-1095.

39. F. O. Wagner, Stable groups, London Mathematical Society Lecture Notes (Cambridge University Press, 1997).

40. M. ZiegleR, A remark on Morley rank, preprint 1997, http://home.mathematik.unifreiburg.de/ziegler/Preprints.html.

41. M. Ziegler, Separably closed fields with Hasse derivations, J. Symbolic Logic 68 (2003), $311-318$. 